TRANSACTIONS OF THE

AMERICAN MATHEMATICAL SOCIETY

Volume 354, Number 4, Pages 1487-1509

S 0002-9947(01)02911-7

Article electronically published on November 30, 2001

\title{
PRODUCT SYSTEMS OVER RIGHT-ANGLED ARTIN SEMIGROUPS
}

\author{
NEAL J. FOWLER AND AIDAN SIMS
}

\begin{abstract}
We build upon Mac Lane's definition of a tensor category to introduce the concept of a product system that takes values in a tensor groupoid $\mathcal{G}$. We show that the existing notions of product systems fit into our categorical framework, as do the $k$-graphs of Kumjian and Pask. We then specialize to product systems over right-angled Artin semigroups; these are semigroups that interpolate between free semigroups and free abelian semigroups. For such a semigroup we characterize all product systems which take values in a given tensor groupoid $\mathcal{G}$. In particular, we obtain necessary and sufficient conditions under which a collection of $k 1$-graphs form the coordinate graphs of a $k$-graph.
\end{abstract}

\section{INTRODUCTION}

Product systems were introduced by Arveson in his study of one-parameter semigroups of endomorphisms (1]). Very roughly, a product system is a family $\left(E_{t}\right)_{t>0}$ of complex Hilbert spaces that is endowed with an associative multiplication such that, for every $s, t>0$, there is a unitary isomorphism $E_{s} \otimes E_{t} \rightarrow E_{s+t}$ which maps the elementary tensor $x \otimes y$ to the product $x y$. The first discrete analogues of these were studied by Dinh in [3], where the parameter $t$ was constrained to take values in the positive cone of a countable dense subgroup of $\mathbb{R}$. Product systems over arbitrary semigroups were introduced by Fowler and Raeburn in 8 , and the first author has continued this line of investigation in [5] and [6]. Although the papers cited above all focus on the $C^{*}$-algebras associated with product systems, here our interest is purely in the algebraic structure of product systems.

This note was inspired by two recent developments. In [7], the notion of a discrete product system was extended to allow for fibers that are right-Hilbert bimodules over a $C^{*}$-algebra, thus opening connections with Pimsner's generalized Cuntz algebras ([15]). Second, in [12] Kumjian and Pask developed the notion of $k$-graphs, and these have much in common with product systems over the semigroup $\mathbb{N}^{k}$. Our first goal is thus to generalize the definition of a product system to encompass these different algebraic structures. We do this in Section 1 by extending Mac Lane's definition of a monoidal category [13, §VII.1] to that of a tensor groupoid $\mathcal{G}$, and by developing the notion of a product system that takes values in $\mathcal{G}$. In addition to recovering as product systems the algebraic structures mentioned above, by considering an abelian group $G$ as a tensor groupoid we also obtain as a product system every 2-cocycle of the underlying semigroup $S$ that takes values in $G$. This suggests that, at least for some tensor groupoids, the set of all product

Received by the editors December 22, 1999 and, in revised form, June 28, 2001.

1991 Mathematics Subject Classification. Primary 20F36; Secondary 18B40, 55N20.

(C)2001 American Mathematical Society 
systems over a semigroup $S$ should possess a natural binary operation akin to the multiplication of 2-cocycles in $Z^{2}(S ; G)$. At the end of Section 1 we show that this is indeed the case: if $\mathcal{G}$ is a tensor groupoid which is symmetric in the sense of [13. $\S X I .1]$, then one can form the internal tensor product of two product systems over $S$ that take values in $\mathcal{G}$. The internal tensor product is associative and well-defined on isomorphism classes of product systems, so these isomorphism classes have the natural structure of a semigroup; we denote this semigroup $H^{2}(S ; \mathcal{G})$. Although this notation suggests the existence of a cohomology theory, at this point we have been unable to place product systems in such a framework.

Section 2 is devoted to constructing and classifying product systems over rightangled Artin semigroups; these are semigroups which interpolate between free semigroups and free abelian semigroups. For a given right-angled Artin semigroup $P$ with generating set $A$, our main result, Theorem 2.1, gives conditions on an $A$-tuple $\left(X_{a}\right)_{a \in A}$ of objects in $\mathcal{G}$ that allow one to construct a product system over $P$ whose fiber over $a \in A$ is $X_{a}$. In Theorem 2.2 we show that our conditions are necessary, and that every product system over $P$ is obtained by our construction. In particular our results allow us to construct and classify $k$-graphs in terms of the coordinate 1-graphs which generate them; see Remark 2.3. In Proposition 2.8 we use our parameterization to determine when two product systems over $P$ are isomorphic, and in Corollary 2.10 we determine the automorphism group of any product system over $P$. We close with a description of the semigroup $H^{2}(P ; \mathcal{G})$ when $\mathcal{G}$ is a symmetric tensor groupoid (Proposition 2.11); when $\mathcal{G}$ is an abelian group $G$, this gives a computation of the second cohomology group $H^{2}(P ; G)$ (Corollary 2.12).

The authors would like to thank the referee for pointing out the connection between our work and Mac Lane's. The first author would also like to thank Alex Kumjian for several helpful discussions on $k$-graphs.

\section{TEnsor Groupoids And PRODUCT Systems}

Let $\mathcal{G}$ be a groupoid, regarded as a small category with inverses. We will write $X \in \mathcal{G}$ to denote that $X$ is an object in $\mathcal{G}$, and $S \in \operatorname{Hom}\left(X_{1}, X_{2}\right)$ or $S: X_{1} \rightarrow X_{2}$ to denote that $S$ is a morphism from $X_{1}$ to $X_{2}$.

We will assume that $\mathcal{G}$ is endowed with the structure of a (relaxed) monoidal category, in the sense of [13, §VII.1]. Thus $\mathcal{G}$ is part of a sextuple $\left\langle\mathcal{G}, \otimes, 1_{\mathcal{G}}, \mathcal{B}, \lambda, \rho\right\rangle$ in which $\otimes$ is a bifunctor $\otimes: \mathcal{G} \times \mathcal{G} \rightarrow \mathcal{G}, 1_{\mathcal{G}}$ is a distinguished object in $\mathcal{G}$, and $\mathcal{B}$, $\lambda$ and $\rho$ are natural isomorphisms

$$
\begin{gathered}
\mathcal{B}=\mathcal{B}_{X_{1}, X_{2}, X_{3}}: X_{1} \otimes\left(X_{2} \otimes X_{3}\right) \rightarrow\left(X_{1} \otimes X_{2}\right) \otimes X_{3}, \\
\lambda=\lambda_{X}: 1_{\mathcal{G}} \otimes X \rightarrow X, \quad \text { and } \rho=\rho_{X}: X \otimes 1_{\mathcal{G}} \rightarrow X,
\end{gathered}
$$

such that $\rho_{1_{\mathcal{G}}}=\lambda_{1_{\mathcal{G}}}: 1_{\mathcal{G}} \otimes 1_{\mathcal{G}} \rightarrow 1_{\mathcal{G}}$, and such that the following two diagrams commute for every $X_{1}, X_{2}, X_{3}, X_{4} \in \mathcal{G}$ :

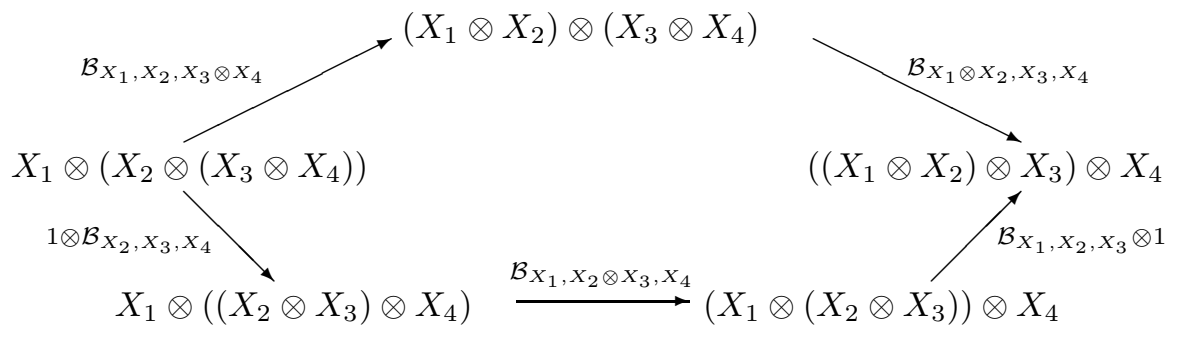


and

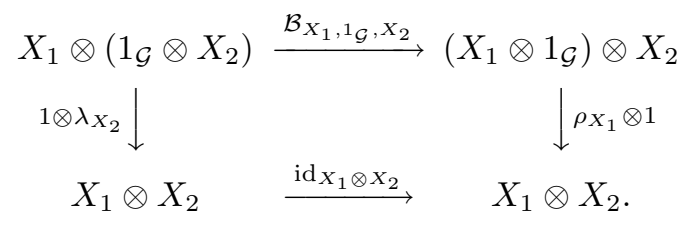

By the corollaries in Sections VII.1 and VII.2 of [13], the canonical isomorphisms supplied by these natural isomorphisms allow us to write expressions such as $X_{1} \otimes$ $\cdots \otimes X_{k}$ without bothering to delineate the order in which adjacent factors should be tensored, and to cancel out any extra factors of $1_{\mathcal{G}}$. We shall take advantage of this notational simplification and make only occasional further references to the natural isomorphisms $\mathcal{B}, \lambda$ and $\rho$.

We have used the symbol $\otimes$ for our bifunctor rather than Mac Lane's more neutral $\square$ since our primary motivating examples truly are tensor products (see Examples 1.5 (2) and (3)). Consequently, we have chosen to expand on Mac Lane's alternative terminology "tensor category" [13, page 252] and refer to $\left\langle\mathcal{G}, \otimes, 1_{\mathcal{G}}, \mathcal{B}, \lambda, \rho\right\rangle$ (or just $\mathcal{G}$ ) as a tensor groupoid.

Definition 1.1. Let $S$ be a countable semigroup, and let $\mathcal{G}$ be a tensor groupoid. A product system over $S$ taking values in $\mathcal{G}$ is a pair $(Y, \alpha)$ in which $Y$ is a collection $\left(Y_{s}\right)_{s \in S}$ of objects in $\mathcal{G}$, and $\alpha$ is a collection $\left(\alpha_{s, t}\right)_{s, t \in S}$ of isomorphisms $\alpha_{s, t}: Y_{s} \otimes$ $Y_{t} \rightarrow Y_{s t}$ such that

$$
\alpha_{r s, t}\left(\alpha_{r, s} \otimes 1_{Y_{t}}\right) \mathcal{B}_{Y_{r}, Y_{s}, Y_{t}}=\alpha_{r, s t}\left(1_{Y_{r}} \otimes \alpha_{s, t}\right) \quad \text { for every } r, s, t \in S .
$$

If $S$ has an identity $e$, we require that $Y_{e}=1_{\mathcal{G}}$, and that, for each $s \in S, \alpha_{e, s}$ and $\alpha_{s, e}$ are implemented by $\lambda_{Y_{s}}$ and $\rho_{Y_{s}}$, respectively.

As alluded to above we will henceforth suppress the natural equivalence $\mathcal{B}$. Equation (1.1) then becomes

$$
\alpha_{r s, t}\left(\alpha_{r, s} \otimes 1_{Y_{t}}\right)=\alpha_{r, s t}\left(1_{Y_{r}} \otimes \alpha_{s, t}\right) \quad \text { for every } r, s, t \in S,
$$

where both sides are regarded as isomorphisms from $Y_{r} \otimes Y_{s} \otimes Y_{t}$ to $Y_{r s t}$; we will write $\alpha_{r, s, t}$ for this isomorphism. More generally:

Notation 1.2. If $k \geq 2$ and $s_{1}, \ldots, s_{k} \in S$, write $\alpha_{s_{1}, \ldots, s_{k}}$ for the isomorphism

$$
Y_{s_{1}} \otimes \cdots \otimes Y_{s_{k}} \rightarrow Y_{s_{1} \cdots s_{k}}
$$

obtained by repeatedly applying appropriate isomorphisms $\alpha_{s, t}$ on adjacent factors. For $s \in S$ we define $\alpha_{s}:=1_{Y_{s}}$.

A moment's thought shows that this notation makes sense: for each way of associating the factors in $Y_{s_{1}} \otimes \cdots \otimes Y_{s_{k}}$ one can apply appropriate isomorphisms $\alpha_{s, t}$ to obtain a morphism with range $Y_{s_{1} \cdots s_{k}}$, and a straightforward inductive argument using the naturality of $\mathcal{B}$ shows that the canonical isomorphisms supplied by $\mathcal{B}$ carry these morphisms into one another.

Definition 1.3. Two product systems $(Y, \alpha)$ and $(Z, \beta)$ are isomorphic if there is a collection $\psi=\left(\psi_{s}\right)_{s \in S}$ of isomorphisms $\psi_{s}: Y_{s} \rightarrow Z_{s}$ such that, for every $s, t \in S$, 
the following diagram commutes:

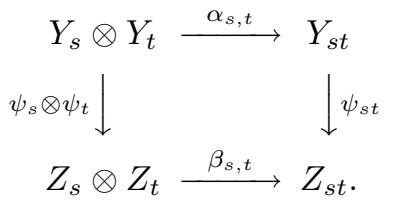

Remark 1.4. It is often useful to not require that the objects of $\mathcal{G}$ form a set. Thus we will sometimes consider structures $\left\langle\mathcal{G}, \otimes, 1_{\mathcal{G}}, \mathcal{B}, \lambda, \rho\right\rangle$ in which $\mathcal{G}$ is a category, all of whose morphisms are invertible. This is merely a convenience; for if $(Y, \alpha)$ is a product system that takes values in such a category $\mathcal{G}$, then $(Y, \alpha)$ takes values in the tensor groupoid $\mathcal{G}^{\prime}$ whose objects are all possible tensor products $Y_{s_{1}} \otimes \cdots \otimes Y_{s_{n}}$.

Examples 1.5. (1) Let $G$ be an abelian group, considered as the morphisms of a groupoid $\mathcal{G}$ with one object. Since $G$ is abelian, $g \otimes h:=g h$ defines a functor $\otimes: \mathcal{G}^{2} \rightarrow \mathcal{G}$, and it is easy to see that this gives $\mathcal{G}$ the structure of a tensor groupoid; the lone object in $\mathcal{G}$ is the identity object $1_{\mathcal{G}}$, and $\lambda$ and $\rho$ are both the identity morphism on $1_{\mathcal{G}}$.

If $(Y, \alpha)$ is a product system over $S$ that takes values in $\mathcal{G}$, then $\alpha$ is a 2-cocycle on $S$ that takes values in $G$. Since $\mathcal{G}$ has but one object, the map $(Y, \alpha) \mapsto \alpha$ is a bijection between such product systems and the group $Z^{2}(S ; G)$. Moreover, cocycles are cohomologous if and only if the corresponding product systems are isomorphic, so there is a canonical bijection between the set of isomorphism classes of product systems and the cohomology group $H^{2}(S ; G)$.

(2) The product systems considered in [3], [4, 8], [5], and [6] can be placed in our categorical framework. We will follow the convention of the latter three references and consider product systems over a monoid $S$, and write $e$ for the identity element in $S$.

Let $\mathcal{G}$ be the category whose objects are nontrivial separable complex Hilbert spaces, and whose morphisms are intertwining unitary isomorphisms. Let $\otimes$ be the usual Hilbert space tensor product, let $1_{\mathcal{G}}=\mathbb{C}$, and let $\mathcal{B}, \lambda$ and $\rho$ be the natural equivalences determined by

$$
\begin{gathered}
\mathcal{B}_{X_{1}, X_{2}, X_{3}}\left(x_{1} \otimes\left(x_{2} \otimes x_{3}\right)\right)=\left(x_{1} \otimes x_{2}\right) \otimes x_{3} \quad \text { for } x_{i} \in X_{i}, \\
\lambda_{X}(z \otimes x)=z x \quad \text { and } \quad \rho_{X}(x \otimes z)=z x
\end{gathered}
$$

for $x \in X$ and $z \in \mathbb{C}$.

Given a product system $(Y, \alpha)$ over $S$ that takes values in $\mathcal{G}$, define

$$
E:=\bigsqcup_{s \in S}\{s\} \times Y_{s},
$$

define $p: E \rightarrow S$ by $p(s, x):=s$, and define multiplication in $E$ by

$$
(s, x)(t, y):=\left(s t, \alpha_{s, t}(x, y)\right) .
$$

Then $E$ is a product system over $S$ in the sense of [8], and it is easy to see that this defines a bijective correspondence between (isomorphism classes of) the two different types of product systems.

We can replace $\mathcal{G}$ with a tensor groupoid by limiting the number of objects. For $n \geq 1$ let $\mathcal{H}_{n}$ be the Hilbert space $\mathbb{C}^{n}$, and let $\mathcal{H}_{\infty}:=\ell^{2}(\mathbb{N})$. Let $\mathcal{G}^{\prime}$ be the tensor groupoid whose objects are all possible Hilbert space tensor products $\mathcal{H}_{n_{1}} \otimes \cdots \otimes \mathcal{H}_{n_{k}}$, and whose morphisms are intertwining unitary operators. Exactly 
as above, every product system over $S$ (in the sense of [8]) corresponds to a product system over $S$ taking values in $\mathcal{G}^{\prime}$.

(3) The product systems studied in [7] can also be placed in our categorical framework. Let $A$ be a $C^{*}$-algebra. A right Hilbert $A$-module is, roughly speaking, a right $A$-module $X_{A}$ which is endowed with an $A$-valued inner product. If $X_{A}$ is endowed with a left action of $A$ by adjointable operators, we call $X$ a right-Hilbert $A-A$ bimodule. (See [15] and [14] for details.)

Let $\mathcal{G}$ be the category in which the objects are right-Hilbert $A-A$ bimodules, and, for objects $X, Y \in \mathcal{G}, \operatorname{Hom}(X, Y)$ is the set of all bimodule isomorphisms $X \rightarrow Y$ that preserve the inner product. As a tensoring functor we use the $A$-balanced internal tensor product (see [14 for details), and then the bimodule ${ }_{A} A_{A}$ serves as the identity object $1_{\mathcal{G}}$. The natural equivalence $\mathcal{B}$ is again given by (1.3), and $\lambda$ and $\rho$ are determined by $\lambda_{X}\left(a \otimes_{A} x\right):=a \cdot x$ and $\rho_{X}\left(x \otimes_{A} a\right)=x \cdot a$ for $x \in X$ and $a \in A$. As in the previous example, product systems that take values in this category correspond to the product systems introduced in [7].

(4) Let $V$ be a countable set. We construct a category $\mathcal{G}$ as follows. The objects in $\mathcal{G}$ are triples $X=\left(E, r_{E}, s_{E}\right)$ in which $E$ is a countable set and $r_{E}$ and $s_{E}$ are functions $E \rightarrow V$; we think of $V$ and $E$ as the vertices and edges of a directed graph, with $r_{E}$ and $s_{E}$ the range and source maps. We will write $r$ and $s$ rather than $r_{E}$ and $s_{E}$ when the domain is clear from context, and we somewhat imprecisely regard $E$ as an object in $\mathcal{G}$. Elements of $\operatorname{Hom}\left(E, E^{\prime}\right)$ are bijections $\varphi: E \rightarrow E^{\prime}$ such that $s=s \circ \varphi$ and $r=r \circ \varphi$.

Define

$$
E_{1} \otimes E_{2}:=\left\{\left(f_{1}, f_{2}\right) \in E_{1} \times E_{2}: r\left(f_{1}\right)=s\left(f_{2}\right)\right\},
$$

with range and source maps

$$
r\left(f_{1}, f_{2}\right):=r\left(f_{2}\right) \quad \text { and } \quad s\left(f_{1}, f_{2}\right):=s\left(f_{1}\right) .
$$

Write $f_{1} \otimes f_{2}$ for the edge $\left(f_{1}, f_{2}\right) \in E_{1} \otimes E_{2}$. For $\varphi_{1} \in \operatorname{Hom}\left(E_{1}, E_{1}^{\prime}\right)$ and $\varphi_{2} \in$ $\operatorname{Hom}\left(E_{2}, E_{2}^{\prime}\right)$, define $\varphi_{1} \otimes \varphi_{2} \in \operatorname{Hom}\left(E_{1} \otimes E_{2}, E_{1}^{\prime} \otimes E_{2}^{\prime}\right)$ by

$$
\varphi_{1} \otimes \varphi_{2}\left(f_{1} \otimes f_{2}\right):=\varphi_{1}\left(f_{1}\right) \otimes \varphi_{2}\left(f_{2}\right) .
$$

Then $\otimes$ is a functor $\mathcal{G}^{2} \rightarrow \mathcal{G}$. Equation (1.3) again defines a natural isomorphism $\mathcal{B}$ between the functors $\otimes \circ\left(\operatorname{id}_{\mathcal{G}} \times \otimes\right)$ and $\otimes \circ\left(\otimes \times \mathrm{id}_{\mathcal{G}}\right)$. We define the identity object $1_{\mathcal{G}}$ to be the triple $\left(V, \mathrm{id}_{V}, \mathrm{id}_{V}\right)$, and define the natural isomorphisms $\lambda$ and $\rho$ by $\lambda_{E}\left(s_{E}(f) \otimes f\right):=f$ and $\rho_{E}\left(f \otimes r_{E}(f)\right):=f$ for all $f \in E$.

When $S$ is a monoid with no nontrivial idempotents, product systems over $S$ that take values in this category are related to the $k$-graphs of Kumjian-Pask [12. To explain the connection, we first recall the definition of a $k$-graph. Let $\Lambda$ be (the morphisms of) a countable small category, and consider $S$ as the morphisms of a small category with one object. A functor $d: \Lambda \rightarrow S$ is said to have the factorization property if for every $\lambda \in \Lambda$ and $t_{1}, t_{2} \in S$ with $d(\lambda)=t_{1} t_{2}$, there are unique elements $\lambda_{1}, \lambda_{2} \in \Lambda$ such that $\lambda=\lambda_{1} \lambda_{2}$ and $d\left(\lambda_{1}\right)=t_{1}, d\left(\lambda_{2}\right)=t_{2}$. When $S=\mathbb{N}^{k}$, such a pair $(\Lambda, d)$ is called a $k$-graph.

Suppose $\Lambda$ is the set of morphisms of a small category with object set $V$, and suppose $d: \Lambda \rightarrow S$ has the factorization property. We think of $(\Lambda, d)$ as a generalized $k$-graph. For each $t \in S$, define $Y_{t}:=d^{-1}(t)$. With range and source maps the reverse of those inherited from $\Lambda$ (that is, $r=$ dom and $s=\operatorname{cod}$ ), $Y_{t}$ becomes an 
object in $\mathcal{G}$. For each $t_{1}, t_{2} \in S$, define $\alpha_{t_{1}, t_{2}}: Y_{t_{1}} \otimes Y_{t_{2}} \rightarrow Y_{t_{1} t_{2}}$ by

$$
\alpha_{t_{1}, t_{2}}\left(f_{1} \otimes f_{2}\right):=f_{1} f_{2} \quad \text { for } f_{1} \in Y_{t_{1}}, f_{2} \in Y_{t_{2}} .
$$

We claim that $(Y, \alpha)$ is a product system over $S$ taking values in $\mathcal{G}$.

To begin with, note that $\alpha_{t_{1}, t_{2}} \in \operatorname{Hom}\left(Y_{t_{1}} \otimes Y_{t_{2}}, Y_{t_{1} t_{2}}\right)$ : each such map clearly preserves the range and source maps, and the factorization property is precisely the condition needed to ensure that $\alpha_{t_{1}, t_{2}}$ is bijective.

Next we will show that $Y_{e}=V$, where each $v \in V$ is identified with $1_{v} \in \Lambda$. Fix $v \in V$. Since $v$ is an idempotent so is $d(v)$, and hence $d(v)=e$; that is, $v \in Y_{e}$. Now fix $\lambda \in Y_{e}$. Since $e^{2}=e$, the factorization property assures us that there are unique elements $\lambda_{1}, \lambda_{2} \in Y_{e}$ such that $\lambda=\lambda_{1} \lambda_{2}$. Since $s(\lambda) \lambda=\lambda=\lambda r(\lambda)$, we conclude that $\lambda=s(\lambda)=r(\lambda) \in V$. Thus $Y_{e}=V$.

Finally, for each $t \in S$ we have

$$
\alpha_{e, t}(s(\lambda) \otimes \lambda)=s(\lambda) \lambda=\lambda=\lambda_{Y_{t}}(s(\lambda) \otimes \lambda) \quad \text { for } \lambda \in Y_{t},
$$

and similarly $\alpha_{t, e}$ is implemented by $\rho_{Y_{t}}$. Thus $(Y, \alpha)$ is a product system over $S$ taking values in $\mathcal{G}$, as claimed.

Conversely, suppose one is given a product system $(Y, \alpha)$ over $S$ taking values in $\mathcal{G}$. Let

$$
\Lambda:=\bigcup_{t \in S}\{t\} \times Y_{t},
$$

and define dom, cod: $\Lambda \rightarrow V$ by $\operatorname{dom}(t, f):=r(f)$ and $\operatorname{cod}(t, f):=s(f)$. Then $\Lambda$ is the set of morphisms of a countable small category with object set $V$, in which morphisms are composed according to

$$
\left(t_{1}, f_{1}\right)\left(t_{2}, f_{2}\right):=\left(t_{1} t_{2}, \alpha_{t_{1}, t_{2}}\left(f_{1} \otimes f_{2}\right)\right) .
$$

Define $d: \Lambda \rightarrow S$ by $d(t, f):=t$. Then $d$ is a functor, and it satisfies the factorization property because each $\alpha_{t_{1}, t_{2}}$ is a bijection.

The procedures outlined above are easily seen to be inverses of one another, and hence product systems over $S$ taking values in $\mathcal{G}$ are essentially the same as generalized $k$-graphs.

It should be pointed out that this example can be regarded as a special case of Example 1.5(3), as follows. Suppose that $(Y, \alpha)$ is a product system over $S$ taking values in $\mathcal{G}$. For each $t \in S$, let $X_{t}$ be the Cuntz-Krieger bimodule associated with the directed graph $Y_{t}$, as in [9] Example 1.2]; $X_{t}$ is the completion of $C_{c}\left(Y_{t}\right)$ with respect to a certain norm defined using the range map for $Y_{t}$. The embeddings $y \in Y_{t} \mapsto \delta_{y} \in X_{t}$ induce isomorphisms $\beta_{s, t}: X_{s} \otimes X_{t} \rightarrow X_{s t}$ that make $(X, \beta)$ into a product system of right-Hilbert $C_{0}(V)-C_{0}(V)$ bimodules.

Symmetric tensor groupoids. We now discuss tensor groupoids which are symmetric in the sense of [13, $\S$ XI.1]. Let $\mathcal{G}$ be a tensor groupoid and let $F: \mathcal{G}^{2} \rightarrow \mathcal{G}^{2}$ be the "flip" functor which interchanges the order of any pair of objects or morphisms (e.g. $\left.F\left(X_{1}, X_{2}\right)=\left(X_{2}, X_{1}\right)\right)$. Suppose there is a natural equivalence $\mathcal{F}$ from $\otimes$ to $\otimes \circ F$; that is, there is a collection of isomorphisms $\mathcal{F}_{X_{1}, X_{2}}: X_{1} \otimes X_{2} \rightarrow X_{2} \otimes X_{1}$ such that

$$
\left(S_{2} \otimes S_{1}\right) \circ \mathcal{F}_{X_{1}, X_{2}}=\mathcal{F}_{Y_{1}, Y_{2}} \circ\left(S_{1} \otimes S_{2}\right)
$$


for all $X_{i}, Y_{i} \in \mathcal{G}$, and all $S_{i} \in \operatorname{Hom}\left(X_{i}, Y_{i}\right)$. Suppose, furthermore, that for every $X_{1}, X_{2}, X_{3} \in \mathcal{G}$, the diagram

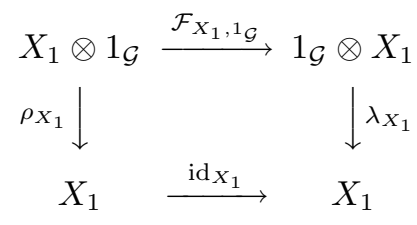

commutes, and that the following two identities hold:

$$
\mathcal{F}_{X_{1}, X_{2}}^{-1}=\mathcal{F}_{X_{2}, X_{1}}
$$

and

$$
\mathcal{F}_{X_{1}, X_{2} \otimes X_{3}}=\left(1_{X_{2}} \otimes \mathcal{F}_{X_{1}, X_{3}}\right)\left(\mathcal{F}_{X_{1}, X_{2}} \otimes 1_{X_{3}}\right) .
$$

Taking inverses in (1.6) and using (1.5), one also has

$$
\mathcal{F}_{X_{1} \otimes X_{2}, X_{3}}=\left(\mathcal{F}_{X_{1}, X_{3}} \otimes 1_{X_{2}}\right)\left(1_{X_{1}} \otimes \mathcal{F}_{X_{2}, X_{3}}\right) .
$$

Following [13, $\S$ XI.1], we call a tensor groupoid $\mathcal{G}$ that admits a natural equivalence $\mathcal{F}$ with these properties a symmetric tensor groupoid.

One can check that this is consistent with Mac Lane's definition of a symmetric monoidal category: our (1.4) corresponds to [13, $\S$ XI.1 (6)], (1.5) corresponds to [13, §XI.1 (8)], and (1.6) and (1.7) correspond to [13, §XI.1 (7)] with the instances of $\mathcal{B}$ suppressed.

Examples 1.6. (1) Let $\mathcal{G}$ be the tensor groupoid associated with an abelian group $G$, as in Examples 1.5(1). Then $\mathcal{G}$ is a symmetric tensor groupoid: $\mathcal{G}^{2}$ has but one object, and assigning the identity element of $G$ to this object gives the desired natural equivalence $\mathcal{F}$.

(2) Let $\mathcal{G}^{\prime}$ be the tensor groupoid introduced in Examples 1.5)(2). For $X_{1}, X_{2} \in \mathcal{G}$, define $\mathcal{F}_{X_{1}, X_{2}}: X_{1} \otimes X_{2} \rightarrow X_{2} \otimes X_{1}$ by

$$
\mathcal{F}_{X_{1}, X_{2}}\left(x_{1} \otimes x_{2}\right):=x_{2} \otimes x_{1} \quad \text { for } x_{i} \in X_{i} .
$$

Then $\mathcal{F}$ is a natural equivalence from $\otimes$ to $\otimes \circ F$, so $\mathcal{G}^{\prime}$ is a symmetric tensor groupoid.

In a symmetric tensor groupoid, one can take tensor products of product systems:

Proposition 1.7. Suppose $(Y, \alpha)$ and $\left(Y^{\prime}, \alpha^{\prime}\right)$ are product systems over semigroups $S$ and $S^{\prime}$, respectively, both taking values in a symmetric tensor groupoid $\mathcal{G}$. For every $\left(s, s^{\prime}\right) \in S \times S^{\prime}$ define

$$
Z_{\left(s, s^{\prime}\right)}:=Y_{s} \otimes Y_{s^{\prime}}^{\prime}
$$

and for every $\left(s, s^{\prime}\right),\left(t, t^{\prime}\right) \in S \times S^{\prime}$ define $\beta_{\left(s, s^{\prime}\right),\left(t, t^{\prime}\right)}: Z_{\left(s, s^{\prime}\right)} \otimes Z_{\left(t, t^{\prime}\right)} \rightarrow Z_{\left(s s^{\prime}, t t^{\prime}\right)}$ by

$$
\beta_{\left(s, s^{\prime}\right),\left(t, t^{\prime}\right)}:=\left(\alpha_{s, t} \otimes \alpha_{s^{\prime}, t^{\prime}}^{\prime}\right)\left(1_{Y_{s}} \otimes \mathcal{F}_{Y_{s^{\prime}}^{\prime}, Y_{t}} \otimes 1_{Y_{t^{\prime}}^{\prime}}\right) .
$$

Then $(Z, \beta)$ is a product system over $S \times S^{\prime}$ taking values in $\mathcal{G}$.

Remark 1.8. We call $(Z, \beta)$ the external tensor product of $(Y, \alpha)$ and $\left(Y^{\prime}, \alpha^{\prime}\right)$. 
Proof. We must show that $\beta$ satisfies the associativity condition (1.2). Suppose $\left(r, r^{\prime}\right),\left(s, s^{\prime}\right),\left(t, t^{\prime}\right) \in S \times S^{\prime}$. Then

$$
\begin{aligned}
\beta_{\left(r, r^{\prime}\right),\left(s t, s^{\prime} t^{\prime}\right)}\left(1_{Z_{\left(r, r^{\prime}\right)}} \otimes \beta_{\left(s, s^{\prime}\right),\left(t, t^{\prime}\right)}\right) & \\
= & \left(\alpha_{r, s t} \otimes \alpha_{r^{\prime}, s^{\prime} t^{\prime}}^{\prime}\right)\left(1_{Y_{r}} \otimes \mathcal{F}_{Y_{r^{\prime}}, Y_{s t}} \otimes 1_{Y_{s^{\prime} t^{\prime}}^{\prime}}\right) \\
& \left(1_{Y_{r} \otimes Y_{r^{\prime}}^{\prime}} \otimes \alpha_{s, t} \otimes \alpha_{s^{\prime}, t^{\prime}}\right)\left(1_{Y_{r} \otimes Y_{r^{\prime}}^{\prime} \otimes Y_{s}} \otimes \mathcal{F}_{Y_{s^{\prime}}^{\prime}, Y_{t}} \otimes 1_{Y_{t^{\prime}}^{\prime}}\right) \\
= & \left(\alpha_{r, s t} \otimes \alpha_{r^{\prime}, s^{\prime} t^{\prime}}^{\prime}\right)\left(1_{Y_{r}} \otimes \alpha_{s, t} \otimes 1_{Y_{r^{\prime}}^{\prime}} \otimes \alpha_{s^{\prime}, t^{\prime}}^{\prime}\right) \\
& \left(1_{Y_{r}} \otimes \mathcal{F}_{Y_{r^{\prime}}^{\prime}, Y_{s} \otimes Y_{t}} \otimes 1_{Y_{s^{\prime}}^{\prime} \otimes Y_{t^{\prime}}^{\prime}}\right)\left(1_{Y_{r} \otimes Y_{r^{\prime}}^{\prime} \otimes Y_{s}} \otimes \mathcal{F}_{Y_{s^{\prime}}^{\prime}, Y_{t}} \otimes 1_{Y_{t^{\prime}}^{\prime}}\right),
\end{aligned}
$$

whereas

$$
\begin{aligned}
\beta_{\left(r s, r^{\prime} s^{\prime}\right),\left(t, t^{\prime}\right)} & \left(\beta_{\left(r, r^{\prime}\right),\left(s, s^{\prime}\right)} \otimes 1_{Z_{\left(t, t^{\prime}\right)}}\right) \\
= & \left(\alpha_{r s, t} \otimes \alpha_{r^{\prime} s^{\prime}, t^{\prime}}^{\prime}\right)\left(1_{Y_{r s}} \otimes \mathcal{F}_{Y_{r^{\prime} s^{\prime}}^{\prime}, Y_{t}} \otimes 1_{Y_{t^{\prime}}^{\prime}}\right) \\
& \left(\alpha_{r, s} \otimes \alpha_{r^{\prime}, s^{\prime}}^{\prime} \otimes 1_{Y_{t} \otimes Y_{t^{\prime}}^{\prime}}\right)\left(1_{Y_{r}} \otimes \mathcal{F}_{Y_{r^{\prime}}^{\prime}, Y_{s}} \otimes 1_{Y_{s^{\prime}}^{\prime} \otimes Y_{t} \otimes Y_{t^{\prime}}^{\prime}}\right) \\
= & \left(\alpha_{r s, t} \otimes \alpha_{r^{\prime} s^{\prime}, t^{\prime}}^{\prime}\right)\left(\alpha_{r, s} \otimes 1_{Y_{t}} \otimes \alpha_{r^{\prime}, s^{\prime}}^{\prime} \otimes 1_{Y_{t^{\prime}}^{\prime}}\right) \\
& \left(1_{Y_{r} \otimes Y_{s}} \otimes \mathcal{F}_{Y_{r^{\prime}}^{\prime} \otimes Y_{s^{\prime}}^{\prime}, Y_{t}} \otimes 1_{Y_{t^{\prime}}^{\prime}}\right)\left(1_{Y_{r}} \otimes \mathcal{F}_{Y_{r^{\prime}}^{\prime}, Y_{s}} \otimes 1_{Y_{s^{\prime}}^{\prime} \otimes Y_{t} \otimes Y_{t^{\prime}}^{\prime}}\right) .
\end{aligned}
$$

Since $\alpha$ and $\alpha^{\prime}$ each satisfy (1.2), the product of the first two factors in (1.8) is equal to the corresponding product in (1.9). Hence it suffices to show that

$$
\left(\mathcal{F}_{Y_{r^{\prime}}^{\prime}, Y_{s} \otimes Y_{t}} \otimes 1_{Y_{s^{\prime}}^{\prime}}\right)\left(1_{Y_{r^{\prime}}^{\prime} \otimes Y_{s}} \otimes \mathcal{F}_{Y_{s^{\prime}}^{\prime}, Y_{t}}\right)=\left(1_{Y_{s}} \otimes \mathcal{F}_{Y_{r^{\prime}}^{\prime} \otimes Y_{s^{\prime}}^{\prime}, Y_{t}}\right)\left(\mathcal{F}_{Y_{r^{\prime}}^{\prime}, Y_{s}} \otimes 1_{Y_{s^{\prime}}^{\prime} \otimes Y_{t}}\right) .
$$

By (1.6), the left-hand side of this equation is equal to

$$
\left(1_{Y_{s}} \otimes \mathcal{F}_{Y_{r^{\prime}}^{\prime}, Y_{t}} \otimes 1_{Y_{s^{\prime}}^{\prime}}\right)\left(\mathcal{F}_{Y_{r^{\prime}}^{\prime}, Y_{s}} \otimes 1_{Y_{t} \otimes Y_{s^{\prime}}^{\prime}}\right)\left(1_{Y_{r^{\prime}}^{\prime} \otimes Y_{s}} \otimes \mathcal{F}_{Y_{s^{\prime}}^{\prime}, Y_{t}}\right),
$$

and by (1.7), the right-hand side is equal to

$$
\left(1_{Y_{s}} \otimes \mathcal{F}_{Y_{r^{\prime}}^{\prime}, Y_{t}} \otimes 1_{Y_{s^{\prime}}^{\prime}}\right)\left(1_{Y_{s} \otimes Y_{r^{\prime}}^{\prime}} \otimes \mathcal{F}_{Y_{s^{\prime}}^{\prime}, Y_{t}}\right)\left(\mathcal{F}_{Y_{r^{\prime}}, Y_{s}} \otimes 1_{Y_{s^{\prime}}^{\prime} \otimes Y_{t}}\right) .
$$

These last two expressions are obviously equal, and the proof is complete.

When $S=S^{\prime}$, one can restrict the external tensor product to the diagonal to obtain another product system over $S$ :

Definition 1.9. Suppose $(Y, \alpha)$ and $(Z, \beta)$ are product systems over $S$ taking values in a symmetric tensor groupoid $\mathcal{G}$. The internal tensor product $(Y, \alpha) \otimes(Z, \beta)$ is the product system $(Y \otimes Z, \alpha \otimes \beta)$ defined by

$$
(Y \otimes Z)_{s}:=Y_{s} \otimes Z_{s} \quad \text { for } s \in S
$$

and

$$
(\alpha \otimes \beta)_{s, t}:=\left(\alpha_{s, t} \otimes \beta_{s, t}\right)\left(1_{Y_{s}} \otimes \mathcal{F}_{Z_{s}, Y_{t}} \otimes 1_{Z_{t}}\right) \quad \text { for } s, t \in S .
$$

Lemma 1.10. The internal tensor product is associative and well-defined on isomorphism classes. 
Proof. Let $(Y, \alpha),(Z, \beta)$ and $(W, \gamma)$ be product systems over $S$ taking values in $\mathcal{G}$, and suppose $s, t \in S$. Making frequent use of (1.6) and (1.7), we calculate

$$
\begin{aligned}
((\alpha \otimes \beta) \otimes \gamma)_{s, t}= & \left((\alpha \otimes \beta)_{s, t} \otimes \gamma_{s, t}\right)\left(1_{Y_{s} \otimes Z_{s}} \otimes \mathcal{F}_{W_{s}, Y_{t} \otimes Z_{t}} \otimes 1_{W_{t}}\right) \\
= & \left(\alpha_{s, t} \otimes \beta_{s, t} \otimes \gamma_{s, t}\right)\left(1_{Y_{s}} \otimes \mathcal{F}_{Z_{s}, Y_{t}} \otimes 1_{Z_{t} \otimes W_{s} \otimes W_{t}}\right) \\
& \left(1_{Y_{s} \otimes Z_{s} \otimes Y_{t}} \otimes \mathcal{F}_{W_{s}, Z_{t}} \otimes 1_{W_{t}}\right)\left(1_{Y_{s} \otimes Z_{s}} \otimes \mathcal{F}_{W_{s}, Y_{t}} \otimes 1_{Z_{t} \otimes W_{t}}\right) \\
= & \left(\alpha_{s, t} \otimes \beta_{s, t} \otimes \gamma_{s, t}\right)\left(1_{Y_{s} \otimes Y_{t} \otimes Z_{s}} \otimes \mathcal{F}_{W_{s}, Z_{t}} \otimes 1_{W_{t}}\right) \\
& \left(1_{Y_{s}} \otimes \mathcal{F}_{Z_{s}, Y_{t}} \otimes 1_{W_{s} \otimes Z_{t} \otimes W_{t}}\right)\left(1_{Y_{s} \otimes Z_{s}} \otimes \mathcal{F}_{W_{s}, Y_{t}} \otimes 1_{Z_{t} \otimes W_{t}}\right) \\
= & \left(\alpha_{s, t} \otimes(\beta \otimes \gamma)_{s, t}\right)\left(1_{Y_{s}} \otimes \mathcal{F}_{Z_{s} \otimes W_{s}, Y_{t}} \otimes 1_{Z_{t} \otimes W_{t}}\right) \\
= & (\alpha \otimes(\beta \otimes \gamma))_{s, t} .
\end{aligned}
$$

This gives associativity.

Now suppose that $\left(\psi_{s}\right)_{s \in S}$ is an isomorphism from $(Y, \alpha)$ to $\left(Y^{\prime}, \alpha^{\prime}\right)$, and that $\left(\varphi_{s}\right)_{s \in S}$ is an isomorphism from $(Z, \beta)$ to $\left(Z^{\prime}, \beta^{\prime}\right)$. For any $s, t \in S$ we have

$$
\begin{aligned}
\left(\psi_{s t}\right. & \left.\otimes \varphi_{s t}\right)(\alpha \otimes \beta)_{s, t} \\
& =\left(\psi_{s t} \otimes \varphi_{s t}\right)\left(\alpha_{s, t} \otimes \beta_{s, t}\right)\left(1_{Y_{s}} \otimes \mathcal{F}_{Z_{s}, Y_{t}} \otimes 1_{Z_{t}}\right) \\
& =\left(\alpha_{s, t}^{\prime} \otimes \beta_{s, t}^{\prime}\right)\left(\psi_{s} \otimes \psi_{t} \otimes \varphi_{s} \otimes \varphi_{t}\right)\left(1_{Y_{s}} \otimes \mathcal{F}_{Z_{s}, Y_{t}} \otimes 1_{Z_{t}}\right) \\
& =\left(\alpha_{s, t}^{\prime} \otimes \beta_{s, t}^{\prime}\right)\left(1_{Y_{s}^{\prime}} \otimes \mathcal{F}_{Z_{s}^{\prime}, Y_{t}^{\prime}} \otimes 1_{Z_{t}^{\prime}}\right)\left(\psi_{s} \otimes \varphi_{s} \otimes \psi_{t} \otimes \varphi_{t}\right) \\
& =\left(\alpha^{\prime} \otimes \beta^{\prime}\right)_{s, t}\left(\psi_{s} \otimes \varphi_{s} \otimes \psi_{t} \otimes \varphi_{t}\right),
\end{aligned}
$$

so $\left(\psi_{s} \otimes \varphi_{s}\right)_{s \in S}$ is an isomorphism from $(Y \otimes Z, \alpha \otimes \beta)$ to $\left(Y^{\prime} \otimes Z^{\prime}, \alpha^{\prime} \otimes \beta^{\prime}\right)$.

Remark 1.11. Essentially the same proofs show that the external tensor product is also associative and well-defined on isomorphism classes of product systems.

Motivated by Examples 1.5(1), we write $Z^{2}(S ; \mathcal{G})$ for the set of product systems over $S$ taking values in $\mathcal{G}$, and $H^{2}(S ; \mathcal{G})$ for the set of isomorphism classes of such product systems. It follows from the previous lemma that when $\mathcal{G}$ is symmetric, the internal tensor product makes both $Z^{2}(S ; \mathcal{G})$ and $H^{2}(S ; \mathcal{G})$ into semigroups.

When $\mathcal{G}$ is the symmetric tensor groupoid associated with an abelian group $G$ (as in Examples 1.5(1) and 1.6(1)), the map $(Y, \alpha) \in Z^{2}(S ; \mathcal{G}) \mapsto \alpha \in Z^{2}(S ; G)$ is an isomorphism of groups, and descends to an isomorphism from $H^{2}(S ; \mathcal{G})$ to $H^{2}(S ; G)$. In the next section we give an explicit description of $H^{2}(S ; \mathcal{G})$ for arbitrary $\mathcal{G}$ in the special case when $S$ is a right-angled Artin semigroup.

\section{Right-Angled Artin semigroups}

Let $\Gamma$ be a (non-directed) graph with countable vertex set $A$. We will assume that $\Gamma$ is simple; that is, that $\Gamma$ has no loops (edges from a vertex to itself) or multiple edges. We write $a \leftrightarrow b$ when $a, b \in A$ are joined by an edge in $\Gamma$.

Let $\mathbb{F}_{A}$ be the free group on $A$, and let $*_{\Gamma} \mathbb{Z}$ be the graph product of $|A|$ copies of $\mathbb{Z}$; that is, $*_{\Gamma} \mathbb{Z}$ is the quotient of $\mathbb{F}_{A}$ by the smallest normal subgroup that contains the commutators $[a, b]$ for which $a \leftrightarrow b$. (See [10] and 11] for details.) Since each of the factors in the graph product is $\mathbb{Z}, *_{\Gamma} \mathbb{Z}$ is a right-angled Artin group. Let $\pi: \mathbb{F}_{A} \rightarrow *_{\Gamma} \mathbb{Z}$ be the canonical quotient map.

Consider the homomorphism $\ell: \mathbb{F}_{A} \rightarrow \mathbb{Z}$ determined by $\ell(a)=1$ for $a \in A$. Since every commutator $[a, b]$ belongs to the kernel of $\ell$, we have $\operatorname{ker} \pi \subseteq \operatorname{ker} \ell$. Thus $\ell$ descends to a homomorphism $*_{\Gamma} \mathbb{Z} \rightarrow \mathbb{Z}$, also denoted $\ell$, which satisfies $\ell(\pi(a))=1$ for each $a \in A$. We call $\ell$ the length function. 
Let $\mathbb{F}_{A}^{+}$be the subsemigroup of $\mathbb{F}_{A}$ generated by $A$. Each element $\mu \in \mathbb{F}_{A}^{+}$can be uniquely written as a word in the alphabet $A$; we denote by $\mu_{i}$ the $i^{\text {th }}$ element of this word, so that $\mu=\mu_{1} \cdots \mu_{\ell(\mu)}$ with $\mu_{1}, \ldots, \mu_{\ell(\mu)} \in A$.

Let $P$ be the subsemigroup of $*_{\Gamma} \mathbb{Z}$ which is the image of $\mathbb{F}_{A}^{+}$under the quotient map $\pi$. We call $P$ a right-angled Artin semigroup. It is worth bearing in mind the following extreme cases: if $\Gamma$ has no edges, then $P$ is the free semigroup $\mathbb{F}_{A}^{+}$, whereas if $\Gamma$ is the complete graph on $A$, then $P$ is free abelian.

The remainder of this note is devoted to constructing and classifying product systems over $P$. Our analysis makes use of a specific section $\delta: P \rightarrow \mathbb{F}_{A}^{+}$of the quotient map $\pi$, called the preferred section. To define it, we fix once and for all a well-ordering of the vertex set $A$. (Since $A$ is countable, this does not require the Axiom of Choice: one can simply enumerate the elements of $A$.) The section $\delta$ is defined recursively, starting with $\delta(\pi(a)):=a$ for each $a \in A$. Suppose $\delta$ has been defined on all words of length at most $k$ for some $k \geq 1$. Fix $t \in P$ of length $k+1$, and use the well-ordering of $A$ to define

$$
a:=\min \left\{\mu_{1}: \mu \in \mathbb{F}_{A}^{+}, \pi(\mu)=t\right\} .
$$

Choose any $\mu \in \mathbb{F}_{A}^{+}$such that $\pi(\mu)=t$ and $\mu_{1}=a$, and express $\mu=\mu_{1} \mu^{\prime}$. Then $t^{\prime}:=\pi\left(\mu^{\prime}\right)$ is independent of the choice of $\mu$ and has length $k$, and we define $\delta(t):=a \delta\left(t^{\prime}\right)$.

Now suppose $\mathcal{G}$ is a tensor groupoid. For our first theorem, fix a collection $\left(X_{a}\right)_{a \in A}$ of objects in $\mathcal{G}$, and define

$$
X_{\mu}:=X_{\mu_{1}} \otimes \cdots \otimes X_{\mu_{\ell(\mu)}} \quad \text { for } \mu \in \mathbb{F}_{A}^{+} .
$$

Write $1_{\mu}$ for the identity morphism on $X_{\mu}$.

Suppose $T=\left(T_{a, b}\right)_{a \leftrightarrow b}$ is a collection of isomorphisms

$$
T_{a, b}: X_{a} \otimes X_{b} \rightarrow X_{b} \otimes X_{a}
$$

such that

$$
T_{a, b}^{-1}=T_{b, a} \quad \text { whenever } a \leftrightarrow b,
$$

and, whenever $a, b$ and $c$ form the vertices of a triangle in $\Gamma$ (i.e., whenever $a \leftrightarrow b$, $b \leftrightarrow c$ and $c \leftrightarrow a$ ), the following hexagonal equation is satisfied:

$$
\left(T_{b, c} \otimes 1_{a}\right)\left(1_{b} \otimes T_{a, c}\right)\left(T_{a, b} \otimes 1_{c}\right)=\left(1_{c} \otimes T_{a, b}\right)\left(T_{a, c} \otimes 1_{b}\right)\left(1_{a} \otimes T_{b, c}\right) .
$$

(Both sides of this equation are isomorphisms $X_{a} \otimes X_{b} \otimes X_{c} \rightarrow X_{c} \otimes X_{b} \otimes X_{a}$.)

Our first theorem asserts that such a collection $T$ is all that is necessary to construct a product system over $P$.

Theorem 2.1. Fix a well-ordering of $A$, and let $\delta: P \rightarrow \mathbb{F}_{A}^{+}$be the corresponding preferred section of the quotient map $\pi: \mathbb{F}_{A}^{+} \rightarrow P$. Then there is a unique product system $(Y, \alpha)=\left(Y^{T}, \alpha^{T}\right)$ over $P$ taking values in the tensor groupoid $\mathcal{G}$ such that

$$
\begin{gathered}
Y_{t}=X_{\delta(t)} \quad \text { for every } t \in P, \\
\alpha_{s, t}=1_{\delta(s t)} \quad \text { if } \delta(s t)=\delta(s) \delta(t), \text { and } \\
\alpha_{\pi(a), \pi(b)}=T_{a, b} \quad \text { if } a \leftrightarrow b \text { and } a>b .
\end{gathered}
$$

Our second theorem asserts that, up to isomorphism, this construction gives all possible product systems over $P$. It also implies that the well-ordering used in Theorem[2.1 does not affect the isomorphism class of the resulting product system. 
Theorem 2.2. Suppose $(Z, \beta)$ is a product system over $P$ which takes values in the tensor groupoid $\mathcal{G}$. Define

$$
X_{a}:=Z_{\pi(a)} \quad \text { for } a \in A
$$

and

$$
T_{a, b}:=\beta_{\pi(b), \pi(a)}^{-1} \beta_{\pi(a), \pi(b)} \quad \text { for every } a, b \in A \text { such that } a \leftrightarrow b .
$$

Then the collection $\left(T_{a, b}\right)_{a \leftrightarrow b}$ satisfies (2.1) and (2.2). Moreover, the corresponding product system $\left(Y^{T}, \alpha^{T}\right)$ given by Theorem 2.1 is isomorphic to $(Z, \beta)$ via an isomorphism $\left(\psi_{s}\right)_{s \in P}$ such that, for each $a \in A, \psi_{\pi(a)}$ is the identity morphism on $Z_{\pi(a)}$

Notice that if $a \leftrightarrow b$, then $\pi(a) \pi(b)=\pi(b) \pi(a)$, and the isomorphisms $\beta_{\pi(a), \pi(b)}$ and $\beta_{\pi(b), \pi(a)}$ each have range $Z_{\pi(a) \pi(b)}$. Thus the equation (2.7) used to define $T_{a, b}$ makes sense.

Remark 2.3. Before proving these theorems, we give an application to the $k$-graphs of Kumjian and Pask. Resume the notation of Examples 1.5(4). Let $d: \Lambda \rightarrow \mathbb{N}^{k}$ be a $k$-graph, and let $V:=d^{-1}(0)$ be the set of objects in $\Lambda$. For each $t \in \mathbb{N}^{k}$, $Y_{t}:=d^{-1}(t)$ is the edge set of a directed graph with vertex set $V$; the range and source maps are the reverse of those inherited from $\Lambda$. Moreover, since $d$ satisfies the factorization property, for every $t_{1}, t_{2} \in \mathbb{N}^{k}$ the map $\alpha_{t_{1}, t_{2}}: Y_{t_{1}} \otimes Y_{t_{2}} \rightarrow Y_{t_{1}+t_{2}}$ defined by $\alpha_{t_{1}, t_{2}}\left(f_{1} \otimes f_{2}\right)=f_{1} f_{2}$ is an isomorphism; composing with $\alpha_{t_{2}, t_{1}}^{-1}$ we see that $Y_{t_{1}} \otimes Y_{t_{2}}$ and $Y_{t_{2}} \otimes Y_{t_{1}}$ are isomorphic.

Given a collection $E_{1}, \ldots, E_{k}$ of countable directed graphs, each with vertex set $V$, which satisfy

$$
E_{i} \otimes E_{j} \cong E_{j} \otimes E_{i} \quad \text { for } 1 \leq i, j \leq k,
$$

one might thus ask if there is a $k$-graph $d: \Lambda \rightarrow \mathbb{N}^{k}$ such that $d^{-1}\left(e_{i}\right)$ is isomorphic to $E_{i}$ for each $i$. (Here $\left\{e_{i}: 1 \leq i \leq k\right\}$ is the canonical basis for $\mathbb{N}^{k}$.) In [12, Section 6], Kumjian and Pask observed that, when $k=2$, any isomorphism $\theta: E_{2} \otimes E_{1} \rightarrow E_{1} \otimes E_{2}$ can be used to construct such a 2-graph. Roughly, the idea is this. For each $t \in \mathbb{N}^{2}$ let

$$
E_{t}:=E_{1}^{\otimes t_{1}} \otimes E_{2}^{\otimes t_{2}}
$$

with the usual range and source maps, and define $\Lambda:=\bigsqcup_{t \in \mathbb{N}^{k}} E_{t}$. One can use $\theta$ in the obvious way to construct isomorphisms $E_{s} \otimes E_{t} \rightarrow E_{s+t}$ for every $s, t \in \mathbb{N}^{2}$, and the resulting binary operation on $\Lambda$ makes it a small category with object set $V$. With $d: \Lambda \rightarrow \mathbb{N}^{k}$ defined by $d(f):=t$ for $f \in E_{t},(\Lambda, d)$ is a $k$-graph.

When $k \geq 3$, things are more complicated. For each pair $(i, j)$ with $1 \leq i<$ $j \leq k$, fix an isomorphism $E_{j} \otimes E_{i} \rightarrow E_{i} \otimes E_{j}$. Somewhat imprecisely, we write $f \otimes g \mapsto g^{\prime} \otimes f^{\prime}$ for each of these isomorphisms, and we also write $g \otimes f \mapsto f^{\prime} \otimes g^{\prime}$ for the inverse maps $E_{i} \otimes E_{j} \rightarrow E_{j} \otimes E_{i}$. Taking $A=\{1, \ldots, k\}$ with its usual ordering, Theorems 2.1 and 2.2 say that the analogue of the construction of 2graphs outlined above yields a $k$-graph if and only if the following condition holds: whenever $1 \leq i<j<l \leq k$, the composite map

$$
\begin{aligned}
f \otimes g \otimes h & \mapsto f \otimes h^{\prime} \otimes g^{\prime} \mapsto h^{\prime \prime} \otimes f^{\prime} \otimes g^{\prime} \mapsto h^{\prime \prime} \otimes g^{\prime \prime} \otimes f^{\prime \prime} \\
& \mapsto g^{\prime \prime \prime} \otimes h^{\prime \prime \prime} \otimes f^{\prime \prime} \mapsto g^{\prime \prime \prime} \otimes f^{\prime \prime \prime} \otimes h^{\prime \prime \prime \prime} \mapsto f^{\prime \prime \prime \prime} \otimes g^{\prime \prime \prime \prime} \otimes h^{\prime \prime \prime \prime}
\end{aligned}
$$

is the identity on $E_{l} \otimes E_{j} \otimes E_{i}$. Moreover, up to isomorphism, every $k$-graph arises in this manner. 
Condition (2.8) holds vacuously when $k=2$, thus reproducing the result of Kumjian and Pask. It also holds when the vertex matrices $M_{i}$ of the directed graphs $E_{i}$ satisfy Robertson and Steger's conditions (H0), (H1a), (H1b) and (H1c) from [16]; that is, if the $M_{i}$ 's are pairwise commuting $\{0,1\}$-matrices such $M_{i} M_{j}$ is a $\{0,1\}$-matrix whenever $i<j$, and $M_{i} M_{j} M_{l}$ is a $\{0,1\}$-matrix whenever $i<$ $j<l$. This is easy to see: under these conditions there are unique isomorphisms $E_{j} \otimes E_{i} \rightarrow E_{i} \otimes E_{j}$, and (2.8) holds since the identity map is the only automorphism of $E_{l} \otimes E_{j} \otimes E_{i}$. See [12, Examples 1.7] for a more direct translation of the RobertsonSteger conditions into $k$-graphs.

Note that our result holds for both finite and infinite $k$, and that one can replace $\mathbb{N}^{k}$ with an arbitrary right-angled Artin semigroup to obtain a more general result.

To prove Theorems 2.1 and 2.2, we need a few preliminary results. First some notation and terminology. Define an action of the symmetric group $S_{k}$ on the words of length $k$ in $\mathbb{F}_{A}^{+}$by

$$
\sigma \mu:=\mu_{\sigma^{-1}(1)} \ldots \mu_{\sigma^{-1}(k)} \quad \text { for } \sigma \in S_{k}, \mu \in \mathbb{F}_{A}^{+}, \ell(\mu)=k .
$$

For $1 \leq i \leq k-1$, let $\tau_{i} \in S_{k}$ be the transposition $(i, i+1)$; we shall omit the dependence on $k$, but this should not cause any confusion.

We call $\tau_{i}$ an allowable transposition for $\mu$ if $\mu_{i} \leftrightarrow \mu_{i+1}$. Note that since $\Gamma$ has no loops, $\tau_{i}$ is not an allowable transposition for $\mu$ when $\mu_{i}=\mu_{i+1}$. We call $\sigma \in S_{k}$ an allowable permutation for $\mu$ if it can be written as a product $\tau_{i_{m}} \cdots \tau_{i_{1}}$ in which $\tau_{i_{j}}$ is an allowable transposition for $\tau_{i_{j-1}} \cdots \tau_{i_{1}} \mu$ for each $j$.

Lemma 2.4. Let $\sigma$ and $\rho$ be allowable permutations for $\mu$. Then:

(1) $\pi(\sigma \mu)=\pi(\mu)$.

(2) If $i<j$ and $\mu_{i} \leftrightarrow \mu_{j}$, then $\sigma(i)<\sigma(j)$.

(3) If $\sigma \mu=\rho \mu$, then $\sigma=\rho$.

Proof. The first assertion follows immediately from the graph product relations upon writing $\sigma$ as a product of allowable transpositions. For (2), suppose $i<j$ and $\mu_{i} \leftrightarrow \mu_{j}$. Since the result is obvious for the identity permutation, we may inductively assume that $\sigma=\tau_{l} \kappa$, where $\kappa$ is an allowable permutation for $\mu$ such that $\kappa(i)<\kappa(j)$ and $\tau_{l}$ is an allowable transposition for $\kappa \mu$. Since $\tau_{l}$ is allowable, we have $(\kappa \mu)_{l} \leftrightarrow(\kappa \mu)_{l+1}$; that is, $\mu_{\kappa^{-1}(l)} \leftrightarrow \mu_{\kappa^{-1}(l+1)}$. Since $\mu_{i} \leftrightarrow \mu_{j}$, this implies that either $\kappa(i) \neq l$ or $\kappa(j) \neq l+1$. From the assumption that $\kappa(i)<\kappa(j)$ we can thus deduce that $\tau_{l} \kappa(i)<\tau_{l} \kappa(j)$, and hence $\sigma(i)<\sigma(j)$.

(3) First suppose $\sigma \mu=\mu$. We claim that $\sigma$ is the identity permutation. If not, then there exists $i$ such that $i<\sigma(i)$. From $\sigma \mu=\mu$ we deduce that $\mu_{\sigma^{m}(i)}=$ $\mu_{\sigma^{m+1}(i)}$, and hence $\mu_{\sigma^{m}(i)} \leftrightarrow \mu_{\sigma^{m+1}(i)}$, for every $m$. Repeated applications of (2) yield the contradiction

$$
i<\sigma(i)<\sigma^{2}(i)<\sigma^{3}(i)<\cdots .
$$

Now suppose $\sigma \mu=\rho \mu$. Then $\rho^{-1} \sigma$ is an allowable permutation for $\mu$ such that $\rho^{-1} \sigma \mu=\mu$, and hence $\sigma=\rho$.

We now make use of a result from [10], which, when formulated in the language we have developed, states that if two elements of $\mathbb{F}_{A}^{+}$have the same image under the quotient map $\pi$, then they are connected by a sequence of allowable transpositions; i.e., one is obtainable from the other by an allowable permutation. (See also [11].) Hence for each $\mu \in \mathbb{F}_{A}^{+}$there is an allowable permutation $\sigma$ for $\mu$ such that $\sigma \mu=$ 
$\delta(\pi(\mu))$. By part (3) of the previous lemma, the permutation $\sigma$ is unique. Thus we define:

Definition 2.5. For each $\mu \in \mathbb{F}_{A}^{+}$, let $\sigma_{\mu}$ be the unique allowable permutation for $\mu$ such that $\sigma_{\mu} \mu=\delta(\pi(\mu))$.

For each permutation $\sigma$ let

$$
\iota(\sigma):=\mid\{(i, j): i<j \text { and } \sigma(i)>\sigma(j)\} \mid,
$$

the number of inversions in $\sigma$.

Lemma 2.6. Let $\mu \in \mathbb{F}_{A}^{+}$.

(1) If $\rho$ is an allowable permutation for $\mu$, then $\sigma_{\rho \mu}=\sigma_{\mu} \rho^{-1}$.

(2) If $\sigma_{\mu}(i)>\sigma_{\mu}(i+1)$, then $\iota\left(\sigma_{\tau_{i} \mu}\right)=\iota\left(\sigma_{\mu}\right)-1$ and $\mu_{i} \leftrightarrow \mu_{i+1}$.

Proof. (1) By Lemma 2.4(1) we have

$$
\sigma_{\mu} \mu=\delta(\pi(\mu))=\delta(\pi(\rho \mu))=\sigma_{\rho \mu}(\rho \mu) .
$$

Since $\sigma_{\rho \mu} \rho$ is an allowable permutation for $\mu,(1)$ now follows from part (3) of Lemma 2.4

The assumption $\sigma_{\mu}(i)>\sigma_{\mu}(i+1)$ implies that $\iota\left(\sigma_{\mu} \tau_{i}\right)=\iota\left(\sigma_{\mu}\right)-1$. The first conclusion of (2) now follows from (1), and the second is an immediate consequence of Lemma 2.4 (2).

The following proposition is our main technical result.

Proposition 2.7. Let $T=\left(T_{a, b}\right)_{a \leftrightarrow b}$ be a family of isomorphisms

$$
T_{a, b}: X_{a} \otimes X_{b} \rightarrow X_{b} \otimes X_{a}
$$

which satisfies (2.1) and 2.2). Fix a well-ordering of the vertex set $A$ and let $\delta: P \rightarrow \mathbb{F}_{A}^{+}$be the corresponding preferred section. Then there is a unique family $\left(U_{\mu}\right)_{\mu \in \mathbb{F}_{A}^{+}}$of isomorphisms $U_{\mu}: X_{\mu} \rightarrow X_{\delta(\pi(\mu))}$ which satisfies

$$
U_{\mu}=1_{\mu} \quad \text { if } \delta(\pi(\mu))=\mu
$$

and

$$
U_{\mu}=U_{\tau_{i} \mu} T_{i}^{\mu} \quad \text { whenever } \mu_{i} \leftrightarrow \mu_{i+1},
$$

where $T_{i}^{\mu}$ is the isomorphism

$$
1_{\mu_{1}} \otimes \cdots \otimes 1_{\mu_{i-1}} \otimes T_{\mu_{i}, \mu_{i+1}} \otimes 1_{\mu_{i+2}} \otimes \cdots \otimes 1_{\mu_{\ell(\mu)}}: X_{\mu} \rightarrow X_{\tau_{i} \mu} .
$$

For this family, we have

$$
U_{\delta(\pi(\mu)) \nu}\left(U_{\mu} \otimes 1_{\nu}\right)=U_{\mu \nu}=U_{\mu \delta(\pi(\nu))}\left(1_{\mu} \otimes U_{\nu}\right) \quad \text { for all } \mu, \nu \in \mathbb{F}_{A}^{+} .
$$

Proof. We begin by recursively defining the family $\left(U_{\mu}\right)_{\mu \in \mathbb{F}_{A}^{+}}$. If $\iota(\mu)=0$, define $U_{\mu}:=1_{\mu}$. Now let $k \geq 0$, and suppose that we have defined $U_{\mu}$ for every $\mu \in \mathbb{F}_{A}^{+}$ such that $\iota\left(\sigma_{\mu}\right) \leq k$. Fix $\mu \in \mathbb{F}_{A}^{+}$such that $\iota\left(\sigma_{\mu}\right)=k+1$, and let

$$
j:=\min \left\{l: \sigma_{\mu}(l)>\sigma_{\mu}(l+1)\right\} .
$$

By Lemma 2.6 (2) we have $\iota\left(\sigma_{\tau_{j} \mu}\right)=\iota\left(\sigma_{\mu}\right)-1=k$ (so that $U_{\tau_{j} \mu}$ is defined) and $\mu_{j} \leftrightarrow \mu_{j+1}$ (so that $T_{j}^{\mu}$ is defined), so we can define $U_{\mu}$ recursively by

$$
U_{\mu}:=U_{\tau_{j} \mu} T_{j}^{\mu} .
$$


If $\delta(\pi(\mu))=\mu$, then $\iota\left(\sigma_{\mu}\right)=0$, so (2.9) holds by definition of $U_{\mu}$. We claim that

$$
U_{\mu}=U_{\tau_{i} \mu} T_{i}^{\mu} \quad \text { whenever } \sigma_{\mu}(i)>\sigma_{\mu}(i+1) ;
$$

again we remark that, by Lemma 2.6 (2), the condition $\sigma_{\mu}(i)>\sigma_{\mu}(i+1)$ ensures that $T_{i}^{\mu}$ is defined. Before verifying (2.12), let us show how it implies (2.10). For this, it suffices to show that (2.10) holds whenever $\mu_{i} \leftrightarrow \mu_{i+1}$ and $\sigma_{\mu}(i)<\sigma_{\mu}(i+1)$. Using Lemma 2.6(1), we compute that

$$
\sigma_{\tau_{i} \mu}(i)=\sigma_{\mu} \tau_{i}(i)=\sigma_{\mu}(i+1)>\sigma_{\mu}(i)=\sigma_{\mu} \tau_{i}(i+1)=\sigma_{\tau_{i} \mu}(i+1) .
$$

Hence we may apply (2.12) to $\tau_{i} \mu$ to deduce that

$$
U_{\tau_{i} \mu}=U_{\mu} T_{i}^{\tau_{i} \mu} .
$$

Composing both sides on the right with $\left(T_{i}^{\tau_{i} \mu}\right)^{-1}=T_{i}^{\mu}$ gives (2.10).

We will verify (2.12) by induction on $\iota\left(\sigma_{\mu}\right)$. If $\iota\left(\sigma_{\mu}\right)=0$, then $\sigma_{\mu}=\mathrm{id}$, and (2.12) holds vacuously. Let $k \geq 1$, and suppose inductively that (2.12) holds whenever $\iota\left(\sigma_{\mu}\right) \leq k-1$. Fix $\mu \in \mathbb{F}_{A}^{+}$with $\iota\left(\sigma_{\mu}\right)=k$, and suppose $i \in\{1, \ldots, \ell(\mu)-1\}$ is such that $\sigma_{\mu}(i)>\sigma_{\mu}(i+1)$. Let

$$
j:=\min \left\{l: \sigma_{\mu}(l)>\sigma_{\mu}(l+1)\right\},
$$

so that by definition $U_{\mu}=U_{\tau_{j} \mu} T_{j}^{\mu}$. Then $j \leq i$, and we consider three cases.

Case 1: $i=j$. Then $U_{\mu}=U_{\tau_{i} \mu} T_{i}^{\mu}$ holds by definition of $U_{\mu}$.

Case 2: $i \geq j+2$. that

By Lemma 2.6] 2) we have $\iota\left(\sigma_{\tau_{i} \mu}\right)=k-1$, and using Lemma 2.6(1) we check

$$
\sigma_{\tau_{i} \mu}(j)=\sigma_{\mu} \tau_{i}(j)=\sigma_{\mu}(j)>\sigma_{\mu}(j+1)=\sigma_{\mu} \tau_{i}(j+1)=\sigma_{\tau_{i} \mu}(j+1) .
$$

Hence we may apply (2.12) to $\tau_{i} \mu$ to obtain

$$
U_{\tau_{i} \mu}=U_{\tau_{j} \tau_{i} \mu} T_{j}^{\tau_{i} \mu}
$$

Similarly,

$$
U_{\tau_{j} \mu}=U_{\tau_{i} \tau_{j} \mu} T_{i}^{\tau_{j} \mu}
$$

Since $i \geq j+2$ we have $\tau_{j} \tau_{i}=\tau_{i} \tau_{j}$. Moreover, with $\nu:=\mu_{1} \cdots \mu_{j-1}, \lambda:=$ $\mu_{j+2} \cdots \mu_{i-1}$ and $\theta:=\mu_{i+2} \cdots \mu_{\ell(\mu)}$, we have

$$
\begin{aligned}
T_{j}^{\tau_{i} \mu} T_{i}^{\mu} & =\left(1_{\nu} \otimes 1_{\mu_{i+1} \mu_{i}} \otimes 1_{\lambda} \otimes T_{\mu_{j}, \mu_{j+1}} \otimes 1_{\theta}\right)\left(1_{\nu} \otimes T_{\mu_{i}, \mu_{i+1}} \otimes 1_{\lambda} \otimes 1_{\mu_{j} \mu_{j+1}} \otimes 1_{\theta}\right) \\
& =\left(1_{\nu} \otimes T_{\mu_{i}, \mu_{i+1}} \otimes 1_{\lambda} \otimes 1_{\mu_{j+1} \mu_{j}} \otimes 1_{\theta}\right)\left(1_{\nu} \otimes 1_{\mu_{i} \mu_{i+1}} \otimes 1_{\lambda} \otimes T_{\mu_{j}, \mu_{j+1}} \otimes 1_{\theta}\right) \\
& =T_{i}^{\tau_{j} \mu} T_{j}^{\mu} .
\end{aligned}
$$

Thus

$$
U_{\mu}=U_{\tau_{j} \mu} T_{j}^{\mu}=U_{\tau_{i} \tau_{j} \mu} T_{i}^{\tau_{j} \mu} T_{j}^{\mu}=U_{\tau_{j} \tau_{i} \mu} T_{j}^{\tau_{i} \mu} T_{i}^{\mu}=U_{\tau_{i} \mu} T_{i}^{\mu},
$$

as required.

Case 3: $i=j+1$.

Lemma 2.6(2) gives $\iota\left(\sigma_{\tau_{j} \mu}\right)=k-1$, and, since $\sigma_{\mu}(j)>\sigma_{\mu}(j+1)>\sigma_{\mu}(j+2)$, we can use Lemma 2.6. (1) to check that

$$
\begin{aligned}
\sigma_{\tau_{j} \mu}(j+1) & =\sigma_{\mu} \tau_{j}(j+1)=\sigma_{\mu}(j) \\
& >\sigma_{\mu}(j+2)=\sigma_{\mu} \tau_{j}(j+2)=\sigma_{\tau_{j} \mu}(j+2) .
\end{aligned}
$$

Hence we may apply (2.12) to $\tau_{j} \mu$ to obtain

$$
U_{\tau_{j} \mu}=U_{\tau_{j+1} \tau_{j} \mu} T_{j+1}^{\tau_{j} \mu}
$$


By Lemma 2.6(2), (2.13) also implies that $\iota\left(\sigma_{\tau_{j+1} \tau_{j}}\right)=\iota\left(\sigma_{\tau_{j} \mu}\right)-1=k-2$, and using Lemma 2.6) (1) we check that

$$
\begin{aligned}
\sigma_{\tau_{j+1} \tau_{j} \mu}(j) & =\sigma_{\mu} \tau_{j} \tau_{j+1}(j)=\sigma_{\mu}(j+1) \\
& >\sigma_{\mu}(j+2)=\sigma_{\mu} \tau_{j} \tau_{j+1}(j+1)=\sigma_{\tau_{j+1} \tau_{j} \mu}(j+1) .
\end{aligned}
$$

Hence we may apply (2.12) to $\tau_{j+1} \tau_{j} \mu$ to obtain

$$
U_{\tau_{j+1} \tau_{j} \mu}=U_{\tau_{j} \tau_{j+1} \tau_{j} \mu} T_{j}^{\tau_{j+1} \tau_{j} \mu} .
$$

Thus

$$
\begin{aligned}
U_{\mu} & =U_{\tau_{j} \mu} T_{j}^{\mu} \\
& =U_{\tau_{j+1} \tau_{j} \mu} T_{j+1}^{\tau_{j} \mu} T_{j}^{\mu} \\
& =U_{\tau_{j} \tau_{j+1} \tau_{j} \mu} T_{j}^{\tau_{j+1} \tau_{j} \mu} T_{j+1}^{\tau_{j} \mu} T_{j}^{\mu} .
\end{aligned}
$$

Since $\sigma_{\mu}(j)>\sigma_{\mu}(j+1)>\sigma_{\mu}(j+2)$, Lemma 2.6 $(2)$ implies that $\mu_{j}, \mu_{j+1}$ and $\mu_{j+2}$ form the vertices of a triangle in $\Gamma$. Using expansions such as

$$
T_{j}^{\mu}=1_{\nu} \otimes\left(T_{\mu_{j}, \mu_{j+1}} \otimes 1_{\mu_{j+2}}\right) \otimes 1_{\theta},
$$

(where $\nu:=\mu_{1} \cdots \mu_{j-1}$ and $\theta:=\mu_{j+3} \cdots \mu_{\ell(\mu)}$ ), the hexagonal equation (2.2) gives

$$
T_{j}^{\tau_{j+1} \tau_{j} \mu} T_{j+1}^{\tau_{j} \mu} T_{j}^{\mu}=T_{j+1}^{\tau_{j} \tau_{j+1} \mu} T_{j}^{\tau_{j+1} \mu} T_{j+1}^{\mu} .
$$

Using this and $\tau_{j} \tau_{j+1} \tau_{j}=\tau_{j+1} \tau_{j} \tau_{j+1}$ in (2.14) gives

$$
U_{\mu}=U_{\tau_{j+1} \tau_{j} \tau_{j+1} \mu} T_{j+1}^{\tau_{j} \tau_{j+1} \mu} T_{j}^{\tau_{j+1} \mu} T_{j+1}^{\mu} .
$$

As above, one now verifies that $\iota\left(\sigma_{\tau_{j+1} \mu}\right)=k-1$ and $\sigma_{\tau_{j+1} \mu}(j)>\sigma_{\tau_{j+1} \mu}(j+1)$, from which (2.12) gives

$$
U_{\tau_{j+1} \mu}=U_{\tau_{j} \tau_{j+1} \mu} T_{j}^{\tau_{j+1} \mu}
$$

One then verifies that $\iota\left(\sigma_{\tau_{j} \tau_{j+1} \mu}\right)=k-2$, and that $\sigma_{\tau_{j} \tau_{j+1} \mu}(j+1)>\sigma_{\tau_{j} \tau_{j+1} \mu}(j+2)$, from which (2.12) gives

$$
U_{\tau_{j} \tau_{j+1} \mu}=U_{\tau_{j+1} \tau_{j} \tau_{j+1} \mu} T_{j+1}^{\tau_{j} \tau_{j+1} \mu} .
$$

Combining these last two equations with (2.15) gives

$$
\begin{aligned}
U_{\mu} & =U_{\tau_{j+1} \tau_{j} \tau_{j+1} \mu} T_{j+1}^{\tau_{j} \tau_{j+1} \mu} T_{j}^{\tau_{j+1} \mu} T_{j+1}^{\mu} \\
& =U_{\tau_{j} \tau_{j+1} \mu} T_{j}^{\tau_{j+1} \mu} T_{j+1}^{\mu} \\
& =U_{\tau_{j+1} \mu} T_{j+1}^{\mu} .
\end{aligned}
$$

This concludes Case 3, and the proof of (2.12) is complete.

For uniqueness, suppose $\left(V_{\mu}\right)_{\mu \in \mathbb{F}_{A}^{+}}$is a different family of isomorphisms $V_{\mu}: X_{\mu} \rightarrow$ $X_{\delta(\pi(\mu))}$ which satisfies (2.9) and (2.10). Choose $\mu$ with $\iota\left(\sigma_{\mu}\right)$ minimal such that $U_{\mu} \neq V_{\mu}$. Since both collections satisfy (2.9) we have $\iota\left(\sigma_{\mu}\right) \geq 1$, and hence there exists $i$ with $\sigma_{\mu}(i)>\sigma_{\mu}(i+1)$. By Lemma 2.6 (2) we have $\iota\left(\sigma_{\tau_{i} \mu}\right)<\iota\left(\sigma_{\mu}\right)$, so by minimality $U_{\tau_{i} \mu}=V_{\tau_{i} \mu}$. Using (2.10) we obtain the contradiction

$$
U_{\mu}=U_{\tau_{i} \mu} T_{i}^{\mu}=V_{\tau_{i} \mu} T_{i}^{\mu}=V_{\mu} .
$$

Thus the collection is unique.

We now verify (2.11). We will prove that

$$
U_{\delta(\pi(\mu)) \nu}\left(U_{\mu} \otimes 1_{\nu}\right)=U_{\mu \nu} \quad \text { for all } \mu, \nu \in \mathbb{F}_{A}^{+} ;
$$


the proof of the other half of (2.11) proceeds in a similar fashion. We verify (2.16) by induction on $\iota\left(\sigma_{\mu}\right)$. If $\iota\left(\sigma_{\mu}\right)=0$, then $\delta(\pi(\mu))=\mu$, and

$$
U_{\delta(\pi(\mu)) \nu}\left(U_{\mu} \otimes 1_{\nu}\right)=U_{\mu \nu}\left(1_{\mu} \otimes 1_{\nu}\right)=U_{\mu \nu} .
$$

Suppose inductively that (2.16) holds whenever $\iota\left(\sigma_{\mu}\right) \leq k-1$. Let $\nu \in \mathbb{F}_{A}^{+}$be arbitrary, and fix $\mu \in \mathbb{F}_{A}^{+}$satisfying $\iota\left(\sigma_{\mu}\right)=k$. Since $k \geq 1$, there exists $i$ such that $\sigma_{\mu}(i)>\sigma_{\mu}(i+1)$, and Lemma 2.6(2) gives $\iota\left(\sigma_{\tau_{i} \mu}\right)=k-1$ and $\mu_{i} \leftrightarrow \mu_{i+1}$. The inductive hypothesis gives that

$$
U_{\delta\left(\pi\left(\tau_{i} \mu\right)\right) \nu}\left(U_{\tau_{i} \mu} \otimes 1_{\nu}\right)=U_{\left(\tau_{i} \mu\right) \nu} .
$$

Since $\pi\left(\tau_{i} \mu\right)=\pi(\mu)$,

$$
\begin{array}{rlrl}
U_{\delta(\pi(\mu)) \nu}\left(U_{\mu} \otimes 1_{\nu}\right) & =U_{\delta\left(\pi\left(\tau_{i} \mu\right)\right) \nu}\left(U_{\tau_{i} \mu} T_{i}^{\mu} \otimes 1_{\nu}\right) & & (\text { by 2.10) } \\
& =U_{\delta\left(\pi\left(\tau_{i} \mu\right)\right) \nu}\left(U_{\tau_{i} \mu} \otimes 1_{\nu}\right)\left(T_{i}^{\mu} \otimes 1_{\nu}\right) & \\
& =U_{\left(\tau_{i} \mu\right) \nu}\left(T_{i}^{\mu} \otimes 1_{\nu}\right) & \\
& =U_{\tau_{i}(\mu \nu)} T_{i}^{\mu \nu} & \\
& =U_{\mu \nu} &
\end{array}
$$

and the proof is complete by induction.

Proof of Theorem 2.1. We begin by proving the existence of a product system $(Y, \alpha)$ which satisfies the conditions of the theorem. The collection $Y$ is determined by (2.3). Let $\left(U_{\mu}\right)_{\mu \in \mathbb{F}_{A}^{+}}$be the family of isomorphisms $U_{\mu}: X_{\mu} \rightarrow X_{\delta(\pi(\mu))}$ given by Proposition 2.7] If $s, t \in P$, then $Y_{s} \otimes Y_{t}=X_{\delta(s)} \otimes X_{\delta(t)}=X_{\delta(s) \delta(t)}$ and $Y_{s t}=$ $X_{\delta(s t)}=X_{\delta(\pi(\delta(s) \delta(t)))}$, so we can define $\alpha_{s, t}: Y_{s} \otimes Y_{t} \rightarrow Y_{s t}$ by

$$
\alpha_{s, t}:=U_{\delta(s) \delta(t)} .
$$

To see that $(Y, \alpha)$ satisfies the associativity condition (1.2), suppose $r, s, t \in P$. Setting $\mu=\delta(r) \delta(s)$ and $\nu=\delta(t)$ in the first part of (2.11) gives

$$
\alpha_{r s, t}\left(\alpha_{r, s} \otimes 1_{Y_{t}}\right)=U_{\delta(r s) \delta(t)}\left(U_{\delta(r) \delta(s)} \otimes 1_{\delta(t)}\right)=U_{\delta(r) \delta(s) \delta(t)},
$$

and setting $\mu=\delta(r)$ and $\nu=\delta(s) \delta(t)$ in the second part of (2.11) gives

$$
\alpha_{r, s t}\left(1_{Y_{r}} \otimes \alpha_{s, t}\right)=U_{\delta(r) \delta(s t)}\left(1_{\delta(r)} \otimes U_{\delta(s) \delta(t)}\right)=U_{\delta(r) \delta(s) \delta(t)} .
$$

Thus (1.2) holds, and $(Y, \alpha)$ is a product system.

To check (2.4), suppose $\delta(s t)=\delta(s) \delta(t)$. By (2.9) we have $U_{\delta(s t)}=1_{\delta(s t)}$, and hence

$$
\alpha_{s, t}=U_{\delta(s) \delta(t)}=U_{\delta(s t)}=1_{\delta(s t)},
$$

giving (2.4). For (2.5), suppose $a, b \in A$ satisfy $a \leftrightarrow b$ and $a>b$. Then $\alpha_{\pi(a), \pi(b)}=$ $U_{\delta(\pi(a)) \delta(\pi(b))}=U_{a b}=U_{b a} T_{a, b}$, where the last equality is by (2.10). But $\delta(\pi(b a))=$ $b a$, so by (2.9) we have $U_{b a}=1_{b a}$, and hence $\alpha_{\pi(a), \pi(b)}=T_{a, b}$, as required.

For uniqueness, we first establish that

$$
\alpha_{s_{1}, \ldots, s_{k}}=U_{\delta\left(s_{1}\right) \cdots \delta\left(s_{k}\right)} \quad \text { for all } k \geq 1 \text { and } s_{1}, \ldots, s_{k} \in P .
$$


(See Notation 1.2.) This equation holds by definition when $k \leq 2$, so suppose inductively that it holds for some $k \geq 2$. Let $s_{1}, \ldots, s_{k+1} \in P$. Then

$$
\begin{array}{rlr}
\alpha_{s_{1}, \ldots, s_{k+1}} & =\alpha_{s_{1} \cdots s_{k}, s_{k+1}}\left(\alpha_{s_{1}, \ldots, s_{k}} \otimes 1_{Y_{s_{k+1}}}\right) & \text { (associativity of } \alpha) \\
& =U_{\delta\left(s_{1} \cdots s_{k}\right) \delta\left(s_{k+1}\right)}\left(U_{\delta\left(s_{1}\right) \cdots \delta\left(s_{k}\right)} \otimes 1_{\delta\left(s_{k+1}\right)}\right) & \text { (induction) } \\
& =U_{\delta\left(s_{1}\right) \cdots \delta\left(s_{k+1}\right)}
\end{array}
$$

with the last equality following from (2.11) by setting $\mu=\delta\left(s_{1}\right) \cdots \delta\left(s_{k}\right)$ and $\nu=$ $\delta\left(s_{k+1}\right)$. Hence (2.18) holds for $k+1$, and inductively for all $k$.

Now suppose $(Y, \beta)$ is another product system over $P$ which satisfies (2.3), (2.4) and 2.5 . Define

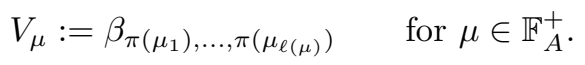

Then $V_{\mu}$ is an isomorphism from $Y_{\pi\left(\mu_{1}\right)} \otimes \cdots \otimes Y_{\pi\left(\mu_{k}\right)}$ to $Y_{\pi(\mu)}$, or, equivalently, from $X_{\mu}$ to $X_{\delta(\pi(\mu))}$. We claim that $\left(V_{\mu}\right)_{\mu \in \mathbb{F}_{A}^{+}}$is the unique family of isomorphisms given by Proposition 2.7 .

We begin by verifying (2.9). Suppose $\delta(\pi(\mu))=\mu$. If $\ell(\mu)=1$, then $V_{\mu}=$ $1_{\mu}$ by definition. Suppose inductively that $V_{\mu}=1_{\mu}$ whenever $\delta(\pi(\mu))=\mu$ and $\ell(\mu) \leq k$ for some $k \geq 1$. Fix $\mu \in \mathbb{F}_{A}^{+}$such that $\delta(\pi(\mu))=\mu$ and $\ell(\mu)=k+1$. Express $\mu=\mu_{1} \nu$. By definition of $\delta(\pi(\mu))$ we have $\delta(\pi(\mu))=\mu_{1} \delta(\pi(\nu))$, and since $\delta(\pi(\mu))=\mu=\mu_{1} \nu$, we deduce that $\delta(\pi(\nu))=\nu$. Since

$$
\delta\left(\pi\left(\mu_{1}\right)\right) \delta(\pi(\nu))=\mu_{1} \nu=\mu=\delta(\pi(\mu))=\delta\left(\pi\left(\mu_{1}\right) \pi(\nu)\right),
$$

(2.4) gives that $\beta_{\pi\left(\mu_{1}\right), \pi(\nu)}=1_{\mu}$. By induction we also have $V_{\nu}=1_{\nu}$, so

$$
V_{\mu}=\beta_{\pi\left(\mu_{1}\right), \pi(\nu)}\left(1_{\pi\left(\mu_{1}\right)} \otimes V_{\nu}\right)=1_{\mu},
$$

as required.

We now verify (2.10). First suppose that $a, b \in A$ satisfy $a \leftrightarrow b$. Then

So

$$
V_{a b}=\beta_{\pi(a), \pi(b)}= \begin{cases}T_{a, b} & \text { if } a>b \\ 1_{a b} & \text { if } a<b\end{cases}
$$

$$
\begin{aligned}
V_{a b} T_{b, a} & = \begin{cases}1_{b a} & \text { if } a>b \\
T_{b, a} & \text { if } a<b\end{cases} \\
& =V_{b a} .
\end{aligned}
$$

Now suppose $\mu \in \mathbb{F}_{A}^{+}$and $\mu_{i} \leftrightarrow \mu_{i+1}$. Express $\mu=\nu \mu_{i} \mu_{i+1} \theta$ with $\nu, \theta \in \mathbb{F}_{A}^{+}$. Then

$$
\begin{aligned}
V_{\tau_{i} \mu} T_{i}^{\mu} & =V_{\tau_{i} \mu}\left(1_{\nu} \otimes T_{\mu_{i}, \mu_{i+1}} \otimes 1_{\theta}\right) \\
& =\beta_{\pi(\nu), \pi\left(\mu_{i+1} \mu_{i}\right), \pi(\theta)}\left(V_{\nu} \otimes V_{\mu_{i+1} \mu_{i}} \otimes V_{\theta}\right)\left(1_{\nu} \otimes T_{\mu_{i}, \mu_{i+1}} \otimes 1_{\theta}\right) \\
& =\beta_{\pi(\nu), \pi\left(\mu_{i} \mu_{i+1}\right), \pi(\theta)}\left(V_{\nu} \otimes V_{\mu_{i} \mu_{i+1}} \otimes V_{\theta}\right) \\
& =V_{\mu},
\end{aligned}
$$

giving 2.10).

By the uniqueness assertion of Proposition 2.7, we have $V_{\mu}=U_{\mu}$ for every $\mu \in \mathbb{F}_{A}^{+}$, which, together with (2.18), gives

$$
\alpha_{\pi\left(\mu_{1}\right), \ldots, \pi\left(\mu_{k}\right)}=\beta_{\pi\left(\mu_{1}\right), \ldots, \pi\left(\mu_{k}\right)} \quad \text { for every } \mu \in \mathbb{F}_{A}^{+} .
$$

Now suppose $s, t \in P$. Let $k:=\ell(s), l:=\ell(t), \mu:=\delta(s)$, and $\nu:=\delta(t)$. Then

$$
\alpha_{s, t}\left(\alpha_{\pi\left(\mu_{1}\right), \ldots, \pi\left(\mu_{k}\right)} \otimes \alpha_{\pi\left(\nu_{1}\right), \ldots, \pi\left(\nu_{l}\right)}\right)=\alpha_{\pi\left(\mu_{1}\right), \ldots, \pi\left(\mu_{k}\right), \pi\left(\nu_{1}\right), \ldots, \pi\left(\nu_{l}\right)}
$$


and

$$
\beta_{s, t}\left(\beta_{\pi\left(\mu_{1}\right), \ldots, \pi\left(\mu_{k}\right)} \otimes \beta_{\pi\left(\nu_{1}\right), \ldots, \pi\left(\nu_{l}\right)}\right)=\beta_{\pi\left(\mu_{1}\right), \ldots, \pi\left(\mu_{k}\right), \pi\left(\nu_{1}\right), \ldots, \pi\left(\nu_{l}\right)},
$$

from which we deduce that $\alpha_{s, t}=\beta_{s, t}$. Thus $(Y, \alpha)$ is the unique product system over $P$ which satisfies (2.3), (2.4) and (2.5).

Proof of Theorem 2.2. It is obvious that (2.1) holds for the family $\left(T_{a, b}\right)_{a \leftrightarrow b}$ defined by (2.7). To verify the hexagonal equation (2.2), suppose $a, b$ and $c$ are the vertices of a triangle in $\Gamma$. We will show that the following equivalent version of (2.2) is satisfied:

$$
\left(T_{b, a} \otimes 1_{c}\right)\left(1_{b} \otimes T_{c, a}\right)\left(T_{c, b} \otimes 1_{a}\right)\left(1_{c} \otimes T_{a, b}\right)\left(T_{a, c} \otimes 1_{b}\right)\left(1_{a} \otimes T_{b, c}\right)=1_{a b c} .
$$

The left-hand side of this equation is

$$
\begin{aligned}
&\left(\beta_{\pi(a), \pi(b)}^{-1}\right.\left.\otimes 1_{c}\right)\left(\beta_{\pi(b), \pi(a)} \otimes 1_{c}\right)\left(1_{b} \otimes \beta_{\pi(a), \pi(c)}^{-1}\right)\left(1_{b} \otimes \beta_{\pi(c), \pi(a)}\right) \\
&\left(\beta_{\pi(b), \pi(c)}^{-1} \otimes 1_{a}\right)\left(\beta_{\pi(c), \pi(b)} \otimes 1_{a}\right)\left(1_{c} \otimes \beta_{\pi(b), \pi(a)}^{-1}\right)\left(1_{c} \otimes \beta_{\pi(a), \pi(b)}\right) \\
&\left(\beta_{\pi(c), \pi(a)}^{-1} \otimes 1_{b}\right)\left(\beta_{\pi(a), \pi(c)} \otimes 1_{b}\right)\left(1_{a} \otimes \beta_{\pi(c), \pi(b)}^{-1}\right)\left(1_{a} \otimes \beta_{\pi(b), \pi(c)}\right),
\end{aligned}
$$

which by five applications of (1.2) simplifies to

$$
\begin{gathered}
\left(\beta_{\pi(a), \pi(b)}^{-1} \otimes 1_{c}\right) \beta_{\pi(b a), \pi(c)}^{-1} \beta_{\pi(b), \pi(a c)} \beta_{\pi(b), \pi(c a)}^{-1} \beta_{\pi(b c), \pi(a)} \beta_{\pi(c b), \pi(a)}^{-1} \beta_{\pi(c), \pi(b a)} \\
\beta_{\pi(c), \pi(a b)}^{-1} \beta_{\pi(c a), \pi(b)} \beta_{\pi(a c), \pi(b)}^{-1} \beta_{\pi(a), \pi(c b)}\left(1_{a} \otimes \beta_{\pi(b), \pi(c)}\right) .
\end{gathered}
$$

Since $\pi(a b)=\pi(b a), \pi(b c)=\pi(c b)$ and $\pi(c a)=\pi(a c)$, this in turn collapses to

$$
\left(\beta_{\pi(a), \pi(b)}^{-1} \otimes 1_{c}\right) \beta_{\pi(a b), \pi(c)}^{-1} \beta_{\pi(a), \pi(b c)}\left(1_{a} \otimes \beta_{\pi(b), \pi(c)}\right),
$$

which by one last application of (1.2) is the identity morphism on $X_{a b c}$, as required.

Let $(Y, \alpha)$ be the product system $\left(Y^{T}, \alpha^{T}\right)$ associated with this collection $T$; that is, $Y_{s}:=X_{\delta(s)}$ and $\alpha_{s, t}:=U_{\delta(s) \delta(t)}$, where $\left(U_{\mu}\right)_{\mu \in \mathbb{F}_{A}^{+}}$is the family of isomorphisms $U_{\mu}: X_{\mu} \rightarrow X_{\delta(\pi(\mu))}$ given by Proposition 2.7 We will define an isomorphism $\psi$ from $(Y, \alpha)$ to $(Z, \beta)$. Fix $s \in P$, let $\mu:=\delta(s)$, and let $k:=\ell(\mu)$. Then

$$
Y_{s}=X_{\delta(s)}=X_{\mu_{1}} \otimes \cdots \otimes X_{\mu_{k}}=Z_{\pi\left(\mu_{1}\right)} \otimes \cdots \otimes Z_{\pi\left(\mu_{k}\right)},
$$

so

$$
\psi_{s}:=\beta_{\pi\left(\mu_{1}\right), \ldots, \pi\left(\mu_{k}\right)}
$$

is an isomorphism from $Y_{s}$ to $Z_{\pi(\mu)}=Z_{s}$. It remains only to show that

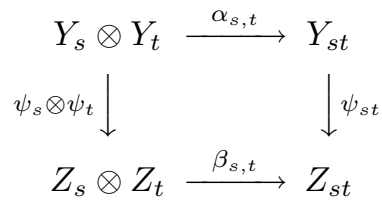

commutes for every $s, t \in P$. Let $\mu=\delta(s)$ as above, let $\nu=\delta(t)$, and let $l:=\ell(\nu)$. Since $\alpha_{s, t}=U_{\mu \nu}$ and $\beta_{s, t}\left(\psi_{s} \otimes \psi_{t}\right)=\beta_{\pi\left(\mu_{1}\right), \ldots, \pi\left(\mu_{k}\right), \pi\left(\nu_{1}\right), \ldots, \pi\left(\nu_{l}\right)}$, it suffices to show that

$$
\beta_{\pi\left(\theta_{1}\right), \ldots, \pi\left(\theta_{m}\right)}=\psi_{\pi(\theta)} U_{\theta} \quad \text { for every } \theta=\theta_{1} \cdots \theta_{m} \in \mathbb{F}_{A}^{+} .
$$

We will establish this equation by induction on $\iota\left(\sigma_{\theta}\right)$. If $\iota\left(\sigma_{\theta}\right)=0$, then $\delta(\pi(\theta))=$ $\theta$, and (2.9) gives $U_{\theta}=1_{\theta}$. Since $\delta(\pi(\theta))=\theta, 2.19$ is then immediate from the definition of $\psi_{\pi(\theta)}$. Suppose inductively that (2.19) holds whenever $\iota\left(\sigma_{\theta}\right) \leq k$ for some $k \geq 0$, and fix $\theta \in \mathbb{F}_{A}^{+}$with $\iota\left(\sigma_{\theta}\right)=k+1$. There exists $i$ such that 
$\sigma_{\theta}(i)>\sigma_{\theta}(i+1)$, and Lemma[2.6 2$)$ gives that $\theta_{i} \leftrightarrow \theta_{i+1}$ and $\iota\left(\sigma_{\tau_{i} \theta}\right)=\iota\left(\sigma_{\theta}\right)-1=k$. By (2.10) and induction we thus have

$$
\psi_{\pi(\theta)} U_{\theta}=\psi_{\pi\left(\tau_{i} \theta\right)} U_{\tau_{i} \theta} T_{i}^{\theta}=\beta_{\pi\left(\theta_{1}\right), \ldots, \pi\left(\theta_{i+1}\right), \pi\left(\theta_{i}\right), \ldots, \pi\left(\theta_{m}\right)} T_{i}^{\theta} .
$$

To further simplify the right-hand side of this equation, let $\mu:=\theta_{1} \cdots \theta_{i-1}$, let $\nu:=\theta_{i+2} \cdots \theta_{m}$, and observe that

$$
\begin{aligned}
& \beta_{\pi\left(\theta_{1}\right), \ldots, \pi\left(\theta_{i+1}\right), \pi\left(\theta_{i}\right), \ldots, \pi\left(\theta_{m}\right)} \\
& \quad=\beta_{\pi(\mu), \pi\left(\theta_{i+1} \theta_{i}\right), \pi(\nu)}\left(\beta_{\pi\left(\theta_{1}\right), \ldots, \pi\left(\theta_{i-1}\right)} \otimes \beta_{\pi\left(\theta_{i+1}\right), \pi\left(\theta_{i}\right)} \otimes \beta_{\pi\left(\theta_{i+2}\right), \ldots, \pi\left(\theta_{m}\right)}\right)
\end{aligned}
$$

and

$$
T_{i}^{\theta}=1_{\mu} \otimes T_{\theta_{i}, \theta_{i+1}} \otimes 1_{\nu} .
$$

By the definition of $T_{\theta_{i}, \theta_{i+1}}$ we have

$$
\beta_{\pi\left(\theta_{i+1}\right), \pi\left(\theta_{i}\right)} T_{\theta_{i}, \theta_{i+1}}=\beta_{\pi\left(\theta_{i}\right), \pi\left(\theta_{i+1}\right)},
$$

so equation (2.20) simplies to

$$
\begin{aligned}
\psi_{\pi(\theta)} U_{\theta} & =\beta_{\pi(\mu), \pi\left(\theta_{i} \theta_{i+1}\right), \pi(\nu)}\left(\beta_{\pi\left(\theta_{1}\right), \ldots, \pi\left(\theta_{i-1}\right)} \otimes \beta_{\pi\left(\theta_{i}\right), \pi\left(\theta_{i+1}\right)} \otimes \beta_{\pi\left(\theta_{i+2}\right), \ldots, \pi\left(\theta_{m}\right)}\right) \\
& =\beta_{\pi\left(\theta_{1}\right), \ldots, \pi\left(\theta_{m}\right)} .
\end{aligned}
$$

This completes the induction, and hence the proof of the theorem.

Proposition 2.8. Suppose $(Y, \alpha)$ and $(Z, \beta)$ are product systems over the rightangled Artin semigroup $P$ which take values in the tensor groupoid $\mathcal{G}$. If $\psi=$ $\left(\psi_{s}\right)_{s \in P}$ is an isomorphism from $(Y, \alpha)$ to $(Z, \beta)$, then defining

$$
\vartheta_{a}:=\psi_{\pi(a)} \quad \text { for } a \in A
$$

gives a collection $\vartheta:=\left(\vartheta_{a}\right)_{a \in A}$ of isomorphisms $\vartheta_{a}: Y_{\pi(a)} \rightarrow Z_{\pi(a)}$ which satisfies

$$
\left(\vartheta_{b} \otimes \vartheta_{a}\right) \alpha_{\pi(b), \pi(a)}^{-1} \alpha_{\pi(a), \pi(b)}=\beta_{\pi(b), \pi(a)}^{-1} \beta_{\pi(a), \pi(b)}\left(\vartheta_{a} \otimes \vartheta_{b}\right)
$$

for every $a, b \in A$ such that $a \leftrightarrow b$. Moreover, given any such collection $\vartheta$, there is a unique isomorphism $\psi:(Y, \alpha) \rightarrow(Z, \beta)$ such that $\psi_{\pi(a)}=\vartheta_{a}$ for every $a \in A$.

Remark 2.9. If $\Gamma$ has no edges, then $P$ is the free semigroup $\mathbb{F}_{A}^{+}$, and Theorem 2.1 associates a product system to each collection $\left(X_{a}\right)_{a \in A}$ of objects in $\mathcal{G}$. Since condition (2.22) is then vacuous, Proposition 2.8 implies that the $A$-tuple of isomorphism classes of the $X_{a}$ 's is a complete isomorphism invariant for product systems over $\mathbb{F}_{A}^{+}$.

Proof of Proposition 2.8. Suppose $\psi=\left(\psi_{s}\right)_{s \in P}$ is an isomorphism from $(Y, \alpha)$ to $(Z, \beta)$; that is, $\psi_{s}$ is an isomorphism $Y_{s} \rightarrow Z_{s}$, and

$$
\psi_{s t} \alpha_{s, t}=\beta_{s, t}\left(\psi_{s} \otimes \psi_{t}\right) \quad \text { for all } s, t \in P .
$$

Applying this equation with $s=\pi(a)$ and $t=\pi(b)$ gives

$$
\beta_{\pi(b), \pi(a)}^{-1} \beta_{\pi(a), \pi(b)}\left(\psi_{\pi(a)} \otimes \psi_{\pi(b)}\right)=\beta_{\pi(b), \pi(a)}^{-1} \psi_{\pi(a) \pi(b)} \alpha_{\pi(a), \pi(b)},
$$

and applying it with $s=\pi(b)$ and $t=\pi(a)$ gives

$$
\left(\psi_{\pi(b)} \otimes \psi_{\pi(a)}\right) \alpha_{\pi(b), \pi(a)}^{-1} \alpha_{\pi(a), \pi(b)}=\beta_{\pi(b), \pi(a)}^{-1} \psi_{\pi(b) \pi(a)} \alpha_{\pi(a), \pi(b)} .
$$

Since $\pi(a) \pi(b)=\pi(b) \pi(a)$, this shows that (2.22) holds for the collection $\vartheta$ defined by (2.21). 
Conversely, suppose we have a collection $\vartheta=\left(\vartheta_{a}\right)_{a \in A}$ of isomorphisms $\vartheta_{a}: Y_{\pi(a)}$ $\rightarrow Z_{\pi(a)}$ which satisfies (2.22). Define

$$
W_{a}:=Y_{\pi(a)} \quad \text { and } \quad X_{a}:=Z_{\pi(a)} \quad \text { for } a \in A
$$

and, for every $a, b \in A$ such that $a \leftrightarrow b$, define

$$
S_{a, b}:=\alpha_{\pi(b), \pi(a)}^{-1} \alpha_{\pi(a), \pi(b)}: W_{a} \otimes W_{b} \rightarrow W_{b} \otimes W_{a}
$$

and

$$
T_{a, b}:=\beta_{\pi(b), \pi(a)}^{-1} \beta_{\pi(a), \pi(b)}: X_{a} \otimes X_{b} \rightarrow X_{b} \otimes X_{a} .
$$

By Theorem 2.2, the collections $S=\left(S_{a, b}\right)_{a \leftrightarrow b}$ and $T=\left(T_{a, b}\right)_{a \leftrightarrow b}$ satisfy equations (2.1) and (2.2), and the product systems $(Y, \alpha)$ and $(Z, \beta)$ are isomorphic to $\left(Y^{S}, \alpha^{S}\right)$ and $\left(Y^{T}, \alpha^{T}\right)$, respectively, via isomorphisms which are the identity on the fibers over $\pi(a)$ for $a \in A$. Hence it suffices to construct an isomorphism $\psi$ from $\left(Y^{S}, \alpha^{S}\right)$ to $\left(Y^{T}, \alpha^{T}\right)$ such that $\psi_{\pi(a)}=\vartheta_{a}$ for every $a \in A$.

We begin by observing that, for each $a \in A, \vartheta_{a}$ is an isomorphism $W_{a} \rightarrow X_{a}$, and that the hypothesis (2.22) can be rewritten as

$$
\left(\vartheta_{b} \otimes \vartheta_{a}\right) S_{a, b}=T_{a, b}\left(\vartheta_{a} \otimes \vartheta_{b}\right) \quad \text { whenever } a \leftrightarrow b .
$$

Define

$$
\vartheta_{\mu}:=\vartheta_{\mu_{1}} \otimes \cdots \otimes \vartheta_{\mu_{\ell(\mu)}}: W_{\mu} \rightarrow X_{\mu} \quad \text { for } \mu \in \mathbb{F}_{A}^{+}
$$

and

$$
\psi_{s}:=\vartheta_{\delta(s)} \quad \text { for } s \in P .
$$

Then $\psi:=\left(\psi_{s}\right)_{s \in P}$ is a collection of isomorphisms $\psi_{s}: Y_{s}^{S} \rightarrow Y_{s}^{T}$. We claim that

$$
\psi_{s t} \alpha_{s, t}^{S}=\alpha_{s, t}^{T}\left(\psi_{s} \otimes \psi_{t}\right) \quad \text { for } s, t \in P
$$

is satisfied, so that $\psi$ is an isomorphism of product systems.

Let $\left(U_{\mu}\right)_{\mu \in \mathbb{F}_{A}^{+}}$and $\left(V_{\mu}\right)_{\mu \in \mathbb{F}_{A}^{+}}$be the families of isomorphisms

$$
U_{\mu}: X_{\mu} \rightarrow X_{\delta(\pi(\mu))} \quad \text { and } \quad V_{\mu}: W_{\mu} \rightarrow W_{\delta(\pi(\mu))}
$$

given by Proposition 2.7, so that

$$
\alpha_{s, t}^{S}=V_{\delta(s) \delta(t)} \quad \text { and } \quad \alpha_{s, t}^{T}=U_{\delta(s) \delta(t)} \quad \text { for } s, t \in P .
$$

The equation (2.24) which we aim to verify can then be rewritten as

$$
\vartheta_{\delta(s t)} V_{\delta(s) \delta(t)}=U_{\delta(s) \delta(t)} \vartheta_{\delta(s) \delta(t)} \quad \text { for all } s, t \in P,
$$

so it suffices to show that

$$
\vartheta_{\delta(\pi(\mu))} V_{\mu}=U_{\mu} \vartheta_{\mu} \quad \text { for all } \mu \in \mathbb{F}_{A}^{+} .
$$

We establish this by induction on $\iota\left(\sigma_{\mu}\right)$. If $\iota\left(\sigma_{\mu}\right)=0$, then $\delta(\pi(\mu))=\mu$, and the equation holds by (2.9). Suppose (2.25) is satisfied whenever $\iota\left(\sigma_{\mu}\right) \leq k-1$ for some $k \geq 1$. Fix $\mu \in \mathbb{F}_{A}^{+}$with $\iota\left(\sigma_{\mu}\right)=k$. Since $k \geq 1$, there exists $i$ such that $\sigma_{\mu}(i)>\sigma_{\mu}(i+1)$, and by Lemma 2.6 (2) we have $\iota\left(\sigma_{\tau_{i} \mu}\right)=\iota\left(\sigma_{\mu}\right)-1=k-1$ and $\mu_{i} \leftrightarrow \mu_{i+1}$. By (2.10) and induction,

$$
\vartheta_{\delta(\pi(\mu))} V_{\mu}=\vartheta_{\delta\left(\pi\left(\tau_{i} \mu\right)\right)} V_{\tau_{i} \mu} S_{i}^{\mu}=U_{\tau_{i} \mu} \vartheta_{\tau_{i} \mu} S_{i}^{\mu} .
$$

Express $\mu=\nu \mu_{i} \mu_{i+1} \theta$ with $\nu, \theta \in \mathbb{F}_{A}^{+}$. Using expansions such as

$$
\vartheta_{\tau_{i} \mu}=\vartheta_{\nu} \otimes\left(\vartheta_{\mu_{i+1}} \otimes \vartheta_{\mu_{i}}\right) \otimes \vartheta_{\theta}
$$


and

$$
S_{i}^{\mu}=1_{\nu} \otimes S_{\mu_{i}, \mu_{i+1}} \otimes 1_{\theta}
$$

it is easy to see that equation (2.23) gives $\vartheta_{\tau_{i} \mu} S_{i}^{\mu}=T_{i}^{\mu} \vartheta_{\mu}$. Using this in (2.26) and applying (2.10) gives

$$
\vartheta_{\delta(\pi(\mu))} V_{\mu}=U_{\tau_{i} \mu} \vartheta_{\tau_{i} \mu} S_{i}^{\mu}=U_{\tau_{i} \mu} T_{i}^{\mu} \vartheta_{\mu}=U_{\mu} \vartheta_{\mu},
$$

thus establishing (2.25).

Using Proposition 2.8 it is easy to characterize the automorphism group of a product system over $P$.

Corollary 2.10. Suppose $(Y, \alpha)$ is a product system over the right-angled Artin semigroup $P$ which takes values in the tensor groupoid $\mathcal{G}$. Then the automorphism group of $(Y, \alpha)$ is isomorphic to the subgroup of $\prod_{a \in A}$ Aut $Y_{\pi(a)}$ consisting of those A-tuples $\left(\vartheta_{a}\right)_{a \in A}$ which satisfy

$$
\alpha_{\pi(a), \pi(b)}\left(\vartheta_{a} \otimes \vartheta_{b}\right) \alpha_{\pi(a), \pi(b)}^{-1}=\alpha_{\pi(b), \pi(a)}\left(\vartheta_{b} \otimes \vartheta_{a}\right) \alpha_{\pi(b), \pi(a)}^{-1}
$$

whenever $a \leftrightarrow b$.

The semigroup $H^{2}(P ; \mathcal{G})$. Let $\mathcal{G}$ be a symmetric tensor groupoid. We now describe the structure of the semigroup $H^{2}(P ; \mathcal{G})$ in terms of the collections $T=$ $\left(T_{a, b}\right)_{a \leftrightarrow b}$ used in Theorem 2.1 to construct product systems $\left(Y^{T}, \alpha^{T}\right)$. Consider the composite map

$$
T \mapsto\left(Y^{T}, \alpha^{T}\right) \in Z^{2}(P ; \mathcal{G}) \mapsto\left[\left(Y^{T}, \alpha^{T}\right)\right] \in H^{2}(P ; \mathcal{G}) .
$$

By Theorem 2.2, this map is surjective and does not depend on the choice of wellordering of the vertex set $A$. The following proposition describes the equivalence relation required on the domain to make the map bijective, and then describes the binary operation on the domain which corresponds to multiplication in $H^{2}(P ; \mathcal{G})$.

Proposition 2.11. Let $\left(W_{a}\right)_{a \in A}$ and $\left(X_{a}\right)_{a \in A}$ be collections of objects in $\mathcal{G}$, and let $S=\left(S_{a, b}\right)_{a \leftrightarrow b}$ and $T=\left(T_{a, b}\right)_{a \leftrightarrow b}$ be collections of isomorphisms

$$
S_{a, b}: W_{a} \otimes W_{b} \rightarrow W_{b} \otimes W_{a} \quad \text { and } \quad T_{a, b}: X_{a} \otimes X_{b} \rightarrow X_{b} \otimes X_{a}
$$

which satisfy conditions (2.1) and (2.2). Then $\left[\left(Y^{S}, \alpha^{S}\right)\right]=\left[\left(Y^{T}, \alpha^{T}\right)\right]$ as elements of $H^{2}(P ; \mathcal{G})$ if and only if there exists a collection $\left(\vartheta_{a}\right)_{a \in A}$ of isomorphisms $\vartheta_{a}: W_{a} \rightarrow X_{a}$ which satisfies

$$
\left(\vartheta_{b} \otimes \vartheta_{a}\right) S_{a, b}=T_{a, b}\left(\vartheta_{a} \otimes \vartheta_{b}\right) \quad \text { whenever } a \leftrightarrow b .
$$

Moreover, multiplication in $H^{2}(P ; \mathcal{G})$ is given by

$$
\left[\left(Y^{S}, \alpha^{S}\right)\right]\left[\left(Y^{T}, \alpha^{T}\right)\right]=\left[\left(Y^{S \otimes T}, \alpha^{S \otimes T}\right)\right],
$$

where $\left((S \otimes T)_{a, b}\right)_{a \leftrightarrow b}$ is the collection of isomorphisms

$$
(S \otimes T)_{a, b}:\left(W_{a} \otimes X_{a}\right) \otimes\left(W_{b} \otimes X_{b}\right) \rightarrow\left(W_{b} \otimes X_{b}\right) \otimes\left(W_{a} \otimes X_{a}\right)
$$

defined by

$$
(S \otimes T)_{a, b}:=\left(1_{W_{b}} \otimes \mathcal{F}_{W_{a}, X_{b}} \otimes 1_{X_{a}}\right)\left(S_{a, b} \otimes T_{a, b}\right)\left(1_{W_{a}} \otimes \mathcal{F}_{X_{a}, W_{b}} \otimes 1_{X_{b}}\right) .
$$


Proof. Since $\left(\alpha_{\pi(b), \pi(a)}^{T}\right)^{-1} \alpha_{\pi(a), \pi(b)}^{T}=T_{a, b}$ whenever $a \leftrightarrow b$, the first assertion follows immediately from Proposition [2.8, For the second, first recall that multiplication in $H^{2}(P ; \mathcal{G})$ is given by the internal tensor product (Definition [1.9), so that

$$
\left[\left(Y^{S}, \alpha^{S}\right)\right]\left[\left(Y^{T}, \alpha^{T}\right)\right]=\left[\left(Y^{S} \otimes Y^{T}, \alpha^{S} \otimes \alpha^{T}\right)\right]
$$

We claim that

$$
(S \otimes T)_{a, b}=\left(\alpha^{S} \otimes \alpha^{T}\right)_{\pi(b), \pi(a)}^{-1}\left(\alpha^{S} \otimes \alpha^{T}\right)_{\pi(a), \pi(b)}
$$

whenever $a \leftrightarrow b$. This will complete the proof, since it then follows from Theorem 2.2 that the collection $\left((S \otimes T)_{a, b}\right)_{a \leftrightarrow b}$ satisfies (2.1) and (2.2), and that $\left(Y^{S \otimes T}, \alpha^{S \otimes T}\right)$ is isomorphic to $\left(Y^{S} \otimes Y^{T}, \alpha^{S} \otimes \alpha^{T}\right)$.

To establish (2.28), first observe that if $a \leftrightarrow b$, then

$$
\begin{aligned}
\left(\alpha_{\pi(b), \pi(a)}^{S}\right. & \left.\otimes \alpha_{\pi(b), \pi(a)}^{T}\right)^{-1}\left(\alpha_{\pi(a), \pi(b)}^{S} \otimes \alpha_{\pi(a), \pi(b)}^{T}\right) \\
& =\left(\left(\alpha_{\pi(b), \pi(a)}^{S}\right)^{-1} \alpha_{\pi(a), \pi(b)}^{S} \otimes\left(\alpha_{\pi(b), \pi(a)}^{T}\right)^{-1} \alpha_{\pi(a), \pi(b)}^{T}\right) \\
& =S_{a, b} \otimes T_{a, b} .
\end{aligned}
$$

Using this and Definition 1.9 we thus have

$$
\begin{aligned}
\left(\alpha^{S} \otimes\right. & \left.\alpha^{T}\right)_{\pi(b), \pi(a)}^{-1}\left(\alpha^{S} \otimes \alpha^{T}\right)_{\pi(a), \pi(b)} \\
= & \left(1_{W_{b}} \otimes \mathcal{F}_{X_{b}, W_{a}} \otimes 1_{X_{a}}\right)^{-1}\left(\alpha_{\pi(b), \pi(a)}^{S} \otimes \alpha_{\pi(b), \pi(a)}^{T}\right)^{-1} \\
& \left(\alpha_{\pi(a), \pi(b)}^{S} \otimes \alpha_{\pi(a), \pi(b)}^{T}\right)\left(1_{W_{a}} \otimes \mathcal{F}_{X_{a}, W_{b}} \otimes 1_{X_{b}}\right) \\
= & \left(1_{W_{b}} \otimes \mathcal{F}_{W_{a}, X_{b}} \otimes 1_{X_{a}}\right)\left(S_{a, b} \otimes T_{a, b}\right)\left(1_{W_{a}} \otimes \mathcal{F}_{X_{a}, W_{b}} \otimes 1_{X_{b}}\right) \\
= & (S \otimes T)_{a, b},
\end{aligned}
$$

giving (2.28).

As a corollary we calculate $H^{2}(P ; G)$ for an arbitrary abelian group $G$. For this, let $E$ be the set of edges in $\Gamma$, and, when $a \leftrightarrow b$, write $e_{a, b}$ for the edge between $a$ and $b$. Note that $e_{a, b}=e_{b, a}$.

Corollary 2.12. Fix a well-ordering of $A$, and let $\delta: P \rightarrow \mathbb{F}_{A}^{+}$be the corresponding preferred section. Then for each function $f: E \rightarrow G$, there is a unique 2-cocycle $\alpha^{f} \in Z^{2}(P ; G)$ which satisfies

$$
\alpha_{s, t}^{f}=1_{G} \quad \text { if } \delta(s t)=\delta(s) \delta(t)
$$

and

$$
\alpha_{\pi(a), \pi(b)}^{f}=f\left(e_{a, b}\right) \quad \text { if } a \leftrightarrow b \text { and } a>b .
$$

The resulting map $f \mapsto\left[\alpha^{f}\right]$ is an isomorphism from $\prod_{e \in E} G$ to $H^{2}(P ; G)$.

Proof. Let $\mathcal{G}$ be the tensor groupoid with one object, morphisms $G$, and tensoring functor $g \otimes h:=g h$ for $g, h \in G$. Given a function $f: E \rightarrow G$, define a collection $\left(T_{a, b}\right)_{a \leftrightarrow b}$ of morphisms by

$$
T_{a, b}:= \begin{cases}f\left(e_{a, b}\right) & \text { if } a<b, \\ f\left(e_{a, b}\right)^{-1} & \text { if } a>b .\end{cases}
$$

Equation (2.1) is obviously satisfied, and the hexagonal equation (2.2) holds since $G$ is abelian. Let $\left(Y^{T}, \alpha^{T}\right) \in Z^{2}(P ; \mathcal{G})$ be the product system given by Theorem 2.1 Then $\alpha^{f}:=\alpha^{T}$ is the unique element of $Z^{2}(P ; G)$ which satisfies (2.29) and (2.30), 
and using this uniqueness property it is easy to see that $f \mapsto\left[\alpha^{f}\right]$ is a group homomorphism from $\prod_{e \in E} G$ to $H^{2}(P ; G)$. Moreover, condition (2.27) in Proposition 2.11 implies that this homomorphism is injective. Since every collection $\left(T_{a, b}\right)$ arises from a function $f: E \rightarrow G$ according to (2.31), and since $T \mapsto\left[\left(Y^{T}, \alpha^{T}\right)\right]$ is surjective, so is $f \mapsto\left[\alpha^{f}\right]$.

Remark 2.13. When $\Gamma$ has no edges, Corollary 2.12 says that the free semigroup $\mathbb{F}_{A}^{+}$has trivial second cohomology. When $\Gamma$ is the complete graph on $A$, it says that $H^{2}\left(\mathbb{N}^{k} ; G\right)$ is isomorphic to the direct product of $\left(\begin{array}{c}k \\ 2\end{array}\right)$ copies of $G$.

\section{REFERENCES}

[1] W. Arveson, Continuous analogues of Fock space, Memoirs Amer. Math. Soc. 80 (1989), No. 409. MR 90f:47061

[2] J. Crisp and M. Laca, On the Toeplitz algebras of right-angled and finite-type Artin groups, J. Austral. Math. Soc., to appear.

[3] H. T. Dinh, Discrete product systems and their $C^{*}$-algebras, J. Funct. Anal. 102 (1991), 1-34. MR 93d:46097

[4] H. T. Dinh, On generalized Cuntz $C^{*}$-algebras, J. Operator Theory 30 (1993), 123-135. MR 95m:46112

[5] N. J. Fowler, Compactly-aligned discrete product systems, and generalizations of $\mathcal{O}_{\infty}$, International J. Math. 10 (1999), No. 6, 721-738. CMP 2000:02

[6] N. J. Fowler, Discrete product systems of finite-dimensional Hilbert spaces, and generalized Cuntz algebras, preprint.

[7] N. J. Fowler, Discrete product systems of Hilbert bimodules, Pacific J. Math., to appear.

[8] N. J. Fowler and I. Raeburn, Discrete product systems and twisted crossed products by semigroups, J. Funct. Anal. 155 (1998), 171-204. MR 99k:46118

[9] N. J. Fowler and I. Raeburn, The Toeplitz algebra of a Hilbert bimodule, Indiana Univ. Math. J. 48 (1999), 155-181. MR 2001b:46093

[10] E. R. Green, Graph products of groups, Thesis, The University of Leeds, 1990.

[11] S. Hermiller and J. Meier, Algorithms and geometry for graph products of groups, J. of Algebra 171 (1995), 230-257. MR 96a:20052

[12] A. Kumjian and D. Pask, Higher rank graph $C^{*}$-algebras, New York J. Math 6 (2000), 1-20. MR 2001b: 46102

[13] S. Mac Lane, Categories for the working mathematician, Graduate Texts in Mathematics, vol. 5, Springer-Verlag, New York, 1998. MR 2001j:18001

[14] P. S. Muhly and B. Solel, Tensor algebras over $C^{*}$-correspondences (representations, dilations, and $C^{*}$-envelopes), J. Funct. Anal. 158 (1998), 389-457. MR 99j:46066

[15] M. V. Pimsner, A class of $C^{*}$-algebras generalizing both Cuntz-Krieger algebras and crossed products by $\mathbb{Z}$, Fields Institute Communications 12 (1997), 189-212. MR 97k:46069

[16] G. Robertson and T. Steger, Affine buildings, tiling systems and higher rank Cuntz-Krieger algebras, J. reine angew. Math. 513 (1999), 115-144. MR 2000j:46109

Department of Mathematics, University of Newcastle, NSW 2308, Australia

Department of Mathematics, University of Newcastle, NSW 2308, Australia 